\title{
Effects of Air Pollution Control on Urban Development Quality in Chinese Cities Based on Spatial Durbin Model
}

\author{
Yanchao Feng, Xiaohong Wang *, Wenchao Du and Jun Liu \\ School of Economics and Management, Harbin Institute of Technology, Harbin 150001, China; \\ m15002182995@163.com (Y.F.); 17B910027@stu.hit.edu.cn (W.D.); liujun6098@163.com (J.L.) \\ * Correspondence: wangxh@hit.edu.cn
}

Received: 19 October 2018; Accepted: 7 December 2018; Published: 11 December 2018

check for updates

\begin{abstract}
With the rapid development of urbanization, industrialization, and motorization, a large number of Chinese cities have been affected by heavy air pollution. In order to promote the development quality of Chinese cities, mixed regulations to control air pollution have been implemented under the lead of government. The principal component analysis and efficacy coefficient method are used to estimate urban development quality, according to the panel data of 285 prefecture-level cities in China over the period 2003-2016. On this basis, the paper uses the spatial Durbin model to study the direct impact and the spatial spillover effect of air pollution control on urban development quality in China. Results show that the control of smoke and dust has improved urban development quality in China, however, the control of sulfur dioxide has led to the decline of urban development quality in China. Furthermore, the impact of air pollution control on urban development quality in the eastern region is of great significance in statistical tests, while the situation in the central and western regions has not passed the test, implying the spatial heterogeneity among different regions. The different effects of air pollution control on urban development quality in different regions also illustrate the consciousness and supervision of local governments' environment protection. Finally, the effects decomposition of the influencing factors based on spatial Durbin model (SDM) also supports the robust findings. Promoting the upgrading of energy consumption structure, raising awareness of environmental protection and supervision, and strengthening cooperation of different regions are suggested. Further recommendations are provided to improve the conceptual design and increase the credibility of our research. Our study not only provides new evidence on the impact of air pollution control on urban development quality in China, but also proposes a new perspective to promote urban development quality in China.
\end{abstract}

Keywords: air pollution control; urban development quality; spatial Durbin model

\section{Introduction}

Over the past decades, the production of energy from various sources (e.g., coal, crude oil, natural gas) has increased rapidly in China, partly caused by the relatively comfortable and prosperous life sought by people, however, along with prosperity, a large number of Chinese cities are affected by heavy air pollution [1]. The unprecedented consequences not only pose challenges to the provision of jobs, housing, and infrastructure, but also exert more pressure on urban land management, spatial equity, and more generally sustainable development [2]. In order to promote the sustainable development of economy and society, the Chinese government has made some active efforts to reduce pollutant emissions and protect the environment. However, despite the government's environmental protection efforts, China's environmental quality seems to be continuously deteriorating, and the 
amount of the emissions of main air pollutants remains persistently high [3]. In recent years, the relationship between air pollution control and economic growth has attracted the attention of many scholars.

With the upgrading of developmental concepts, urban development quality has been transformed from the initial pursuit of urbanization rate or rapid economic growth to the pursuit of sustainable development of economy, society, and environment [4-7]. Characterized by the rise of central business districts (CBDs) for advanced business services, newly formed production centers in suburban areas, and spatially segregated neighborhoods dividing the rich and the poor, the unique patterns of urban development in China has occurred at an unprecedented rate [8-13]. However, the massive construction boom across the whole nation in recent years has left abundant housing, factories, commercial facilities, and new urban districts with incredibly and inexcusably low occupancy rates, known as 'ghost cities/towns' [14]. Therefore, ignoring social welfare and environmental improvement, the immense increase of urban built-up areas or rapid economic growth can not reflect urban development quality comprehensively [15]. Given the fact that enormous regional disparities in levels of development and urbanization across China, it is very necessary to use nationally representative data to further study urban development quality [16].

Nevertheless, there are few studies that explore the global characteristics and the driving factors of urban development quality in China, especially the impact of air pollution control on urban development quality has not been comprehensively and thoroughly examined. Moreover, existing literature overemphasizes the importance of air pollution control on the economic dimension of urban development quality, while the subject of the social and the environmental dimension of urban development quality is particularly under-researched $[17,18]$. Therefore, more sophisticated studies are needed to examine the impact of air pollution control on urban development quality, which is of great and practical significance for guiding the adjustment and optimization of existing air pollution control policies in different regions. Most researchers, academics, and practitioners took it for granted that air pollution control would be effective, and air pollution control would naturally lead to the reduction of environmental pollution and the improvement of environmental quality [19-22]. However, this perception might be misleading. For different objects, samples and methods, the cost and effect of air pollution control are not always consistent [23-26]. Moreover, there is no literature on exploring the impact of air pollution control on the quality of regional urban development. Furthermore, the level of urban development quality and the degree of air pollution control vary in the eastern, central, and western China [16]. Hence, it is necessary to investigate the impact of air pollution control on urban development quality across different regions in China.

As mentioned above, previous related literature is insufficient. To analyze the effects of air pollution control on urban development quality in different regions and reveal the spatial effect of air pollution control on regional urban development quality, this paper has made following contributions to knowledge. Firstly, the effective mechanisms of diversified air pollution control are different. Based on the difference between pre-treatment and post-treatment strategies, air pollution control is classified into two types such as energy saving air pollution control and emission reduction air pollution control. Based on the differences of industrial air pollutants, the energy saving type is measured by the emission of sulfur dioxide per GDP and the smoke and dust per GDP; correspondingly, the emission reduction type is measured by the removal rate of sulfur dioxide and smoke and dust. Secondly, environmental problems are characterized as different regions due to the imbalance of urban development quality. Therefore, in this paper, China is divided into three classical regions: the eastern region, the central region and the western region, to explore the regional difference of the impact of air pollution control on urban development quality. Lastly, the spatial Durbin model is introduced to examine the spatial dependence of air pollution control on urban development quality in China, which is conductive to revising policies of environmental protection for regional government. 


\section{Methodology}

Tobler's first law of geography indicates that all phenomena in space are linked, but the connection intensity is stronger at near distances versus long distances [27]. This law well represents impacts of air pollution control on urban development quality because environmental pollution has strong trans-regional and agglomerate character. In other words, air pollution control and urban development quality show significant spatial dependence. Consequently, we select the spatial Durbin model (which allows testing the existence of both endogenous and exogenous interactions) to investigate the impacts of air pollution control on urban development quality in Chinese cities [28]. Actually, the spatial regression models are frequently applied to economic, environmental, and ecological modeling [29].

\subsection{Spatial Durbin Model}

This study aims to investigate the direct and spillover effects of air pollution control on urban development quality in Chinese cities. Spatial Durbin model (SDM) can examine the influence of the dependent variable affected by the local area variables, as well as the dependent and independent variables in neighboring areas, which is a general form of the spatial lag model (SLM) and spatial error model (SEM). In this way, the spatial Durbin model (SDM) is more suitable for the objectives of this study [30-32]. Its basic form is:

$$
Y_{i t}=\rho \sum_{j=1}^{N} w_{i j} Y_{j t}+\beta X_{i t}+\theta \sum_{j=1}^{N} w_{i j} X_{j t}+\mu+\varepsilon_{i t}
$$

where $Y_{i t}$ is the dependent variable in city $i$ at year $t ; w_{i j}$ corresponds to the spatial connectivity assigned to city $j$ by city $i(j \neq i) ; \rho$ is the spatial parameter of interest, which reflects the endogenous spatial interaction between city $i$ and its neighboring cities; $\beta$ is a vector of the coefficients of the explanatory variables; $X_{i t}$ is the explanatory variables of city $i$ that explains its urban development quality; $\theta$ reflects exogenous interaction effects, which creates an average of explanatory variable values from neighboring cities which are added to the set of conventional explanatory variables; the matrix $w_{i j} X_{j t}$ denotes the spatial lag effects associated with explanatory variables; $\mu$ denotes the random-effects or the time and city fixed effects; and $\varepsilon_{i t}$ represents an error term uncorrelated with the explanatory variables across cities and over time, which is assumed to be normally distributed.

Based on Equation (1), the spatial Durbin model (SDM) of the impact of air pollution control on urban development quality is as follows:

$$
\begin{aligned}
& \ln U D Q_{i t}=\rho \sum_{j=1}^{N} w_{i j} \ln U D Q_{i t}+\beta_{1} \ln E S_{1, i t}+\beta_{2} \ln E S_{2, i t}+\beta_{3} \ln E R_{1, i t}+\beta_{4} \ln E R_{2, i t} \\
& +\beta_{5} \ln L F_{i t}+\beta_{6} \ln F D_{i t}+\beta_{7} \ln H C_{i t}+\beta_{8} \ln F D I_{i t}+\beta_{9} \ln I U_{i t}+\theta_{1} \sum_{j=1}^{N} w_{i j} \ln E S_{1, i t} \\
& +\theta_{2} \sum_{j=1}^{N} w_{i j} \ln E S_{2, i t}+\theta_{3} \sum_{j=1}^{N} w_{i j} \ln E R_{1, i t}+\theta_{4} \sum_{j=1}^{N} w_{i j} \ln E R_{2, i t}+\theta_{5} \sum_{j=1}^{N} w_{i j} \ln L F_{i t} \\
& +\theta_{6} \sum_{j=1}^{N} w_{i j} \ln F D_{i t}+\theta_{7} \sum_{j=1}^{N} w_{i j} \ln H C_{i t}+\theta_{8} \sum_{j=1}^{N} w_{i j} \ln F D I_{i t}+\theta_{9} \sum_{j=1}^{N} w_{i j} \ln I U_{i t} \\
& +\mu+\varepsilon_{i t}
\end{aligned}
$$

where $U D Q$ indicates urban development quality; $E S_{1}, E S_{2}, E S_{3}, E S_{4}$ indicate the emission intensity of sulfur dioxide, the emission intensity of smoke and dust, the removal rate of sulfur dioxide and the removal rate of smoke and dust respectively; $L F$ indicates the shares of land leasing revenue in GDP; $F D$ indicates the shares of both deposits and loans in GDP; HC indicates the number of college students per 10,000 people; FDI indicates the shares of foreign direct investment in GDP; and IU indicates the shares of the value of the tertiary industries in the value of the secondary industries. 
To avoid bias caused by the coefficient estimate of the explanatory variable, the total effect should be divided into a direct and an indirect effect by using the partial derivative method $[30,31]$, and the SDM can be transferred as follows:

$$
Y_{t}=\left[(I-\rho w)^{-1}\left(\beta X_{t}+\theta w X_{t}\right)\right]+(I-\rho w)^{-1} \varepsilon_{t}
$$

The partial differential equation matrix for the $k$ explanatory variable is as follows:

$$
\left[\frac{\partial Y}{\partial X_{1 k}}, \frac{\partial Y}{\partial X_{2 k}}, \cdots \frac{\partial Y}{\partial X_{N k}}\right]_{t}=(I-\rho w)^{-1}\left[\begin{array}{lccc}
\beta_{k} & w_{12} \theta_{k} & \cdots & w_{1 N} \theta_{k} \\
w_{21} \theta_{k} & \beta_{k} & \cdots & w_{2 N} \theta_{k} \\
\vdots & \vdots & \ddots & \vdots \\
w_{N 1} \theta_{k} & w_{N 1} \theta_{k} & \cdots & \beta_{k}
\end{array}\right]
$$

where the average value of the diagonal elements represents the direct effect, the average value of the non-diagonal elements represents the indirect effect (spatial spillover effect), and the sum of the direct effect and the indirect effect is the total effect. Due to the existence of spatial dependence and time inertia, there will be spatial feedback effects on urban development between a certain city and the surrounding cities, resulting in a certain deviation between the decomposition results and the regression results of the spatial Durbin model (SDM) [28-31].

\subsection{Spatial Weight Matrix}

Spatial weight matrix $\left(w_{i j}\right)$ is the core element of spatial panel data models. Two types of spatial weight matrix (the squared term of inverse distance matrix $\left(w_{i j}^{1}\right)$ and the squared term of inverse distance and economic-based matrix $\left(w_{i j}^{2}\right)$ ) are adopted in this study to reflect two different spatial relations, which is defined as follows.

$$
\begin{gathered}
w_{i j}^{1}=\left\{\begin{array}{l}
0, i=j \\
\frac{1}{\left(d_{i j}\right)^{2}}, i \neq j
\end{array}\right. \\
g=\operatorname{diag}\left(\overline{G D P_{1}} / \overline{G D P}, \overline{G D P_{2}} / \overline{G D P}, \ldots \overline{G D P_{N}} / \overline{G D P}\right) \\
w_{i j}^{2}=\left\{\begin{array}{l}
0, i=j \\
\frac{g}{\left(d_{i j}\right)^{2}}, i \neq j
\end{array}\right.
\end{gathered}
$$

where $d_{i j}$ is the greater-circle distance calculated based on the longitude and latitude between city $i$ and city $j$, and $w_{i j}^{1}$ takes into account relations of all cities, which allows for testing all-with-all interactions in the whole territory; $\overline{G D P_{i}}$ and $\overline{G D P}$ present, respectively, the average GDP of city $i$ and all cities during the study periods, and $w_{i j}^{2}$, which is set as all Chinese cities, are inter-connected in terms of both geographical and economic factors. We normalized the two spatial weight matrix to have row-sums of one and main diagonal elements of zero.

\section{Data and Variables}

According to the administrative level, Chinese cities can be divided into prefecture-level cities and county-level cities. Under the administrative division system, a prefecture-level city contains municipal districts and other units, such as county-level cities, counties, and towns. As for the concept of a city, a prefecture-level city in China often refers to the municipal districts, which resembles Western cities [33]. However, a county-level city does not have a clear central urban area and usually contains a large proportion of non-urbanized areas. Therefore, we choose prefecture-level cities as samples for this study. Due to data unavailability (some regions including Taiwan, Hong Kong, and Macau are excluded temporally due to unavailability, some cities have experienced administrative division 
adjustments in the past decade, and other cities have data missing for certain years), a panel data set on 285 prefecture-level cities (see Appendix A) over the period of 2003-2016 has been used (Figure 1).

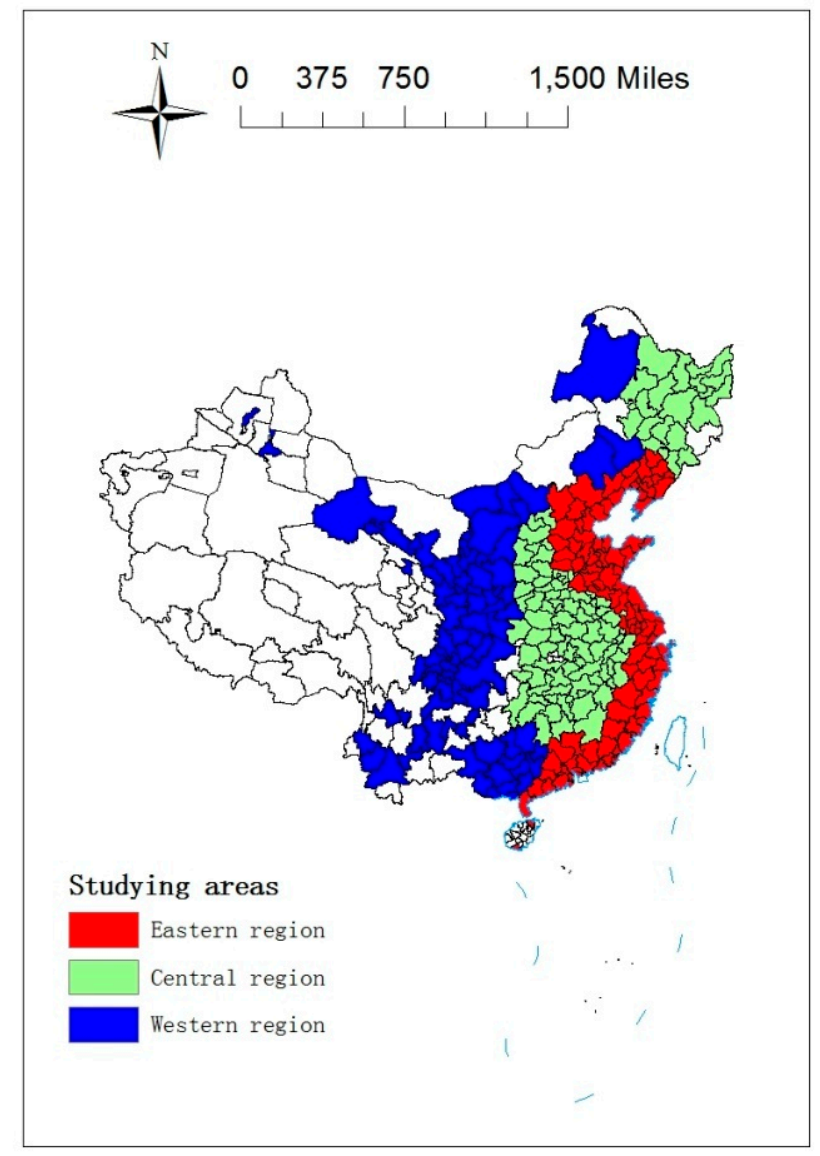

Figure 1. Studying areas.

\subsection{Dependent Variable}

To measure urban development quality comprehensively and accurately, 15 indexes relevant to the three dimensions (economic, social and environmental) should be considered as much as possible within the range of data availability (see Appendix B). To eliminate the effect of dimension and magnitude factors, 15 indexes of urban development quality in each year are normalized as follows:

$$
y_{j i}^{*}=\frac{y_{j i}-\mu_{j i}}{\sqrt{\sigma_{j i}}}
$$

where $y_{j i}^{*}$ is the normalized value of the related statistical factor $j$ in the city $i$, between 0 and $1 ; y_{j i}$ is the original value of the related statistic factor $j$ in the city $i$ of the 285 prefecture-level cities in each year; $\mu_{j i}$ is the average value of the related statistic factor $j$ in the city $i$ of the 285 prefecture-level cities in each year; and $\sigma_{j i}$ is the variance value of the related statistic factor $j$ in the city $i$ of the 285 prefecture-level cities in each year. After normalizing the original data with this equation, the normalized data variance is 1 , and the average value is 0 .

The most commonly used multivariate statistical analysis method is Principal Component Analysis (PCA), which can select several important variables to reduce the number of factors by using linear transformation [34]. Application of the varimax rotation of the normalized component loading allows us to obtain a clear system by maximizing component load differences and eliminating invalid components [35]. 
The model, with the sample set, is as follows:

$$
Y=\left[\begin{array}{lclc}
y_{11} & y_{12} & \cdots & y_{1 p} \\
y_{21} & y_{22} & \cdots & y_{2 p} \\
\vdots & \vdots & \ddots & \vdots \\
y_{n 1} & y_{n 2} & \cdots & y_{n p}
\end{array}\right]_{n \times p}
$$

where $n$ is the number of samples and $p$ is the number of factors.

The principle component analysis of matrix (8) can be combined into $p$ synthesis factors $y_{1}, y_{2}, \cdots y_{p}$, as follows:

$$
\left\{\begin{array}{l}
y_{1}=c_{11} y_{11}+c_{12} y_{12}+\cdots+c_{1 p} x_{p} \\
y_{2}=c_{21} y_{21}+c_{22} y_{22}+\cdots+c_{2 p} x_{p} \\
\cdots \cdots \\
y_{p}=c_{p 1} y_{11}+c_{p 2} y_{12}+\cdots+c_{p p} x_{p}
\end{array}\right.
$$

where $c_{k 1}^{2}+c_{k 2}^{2}+\cdots+c_{k p}^{2}=1(k=1,2, \cdots p)$, and the comprehensive index factors $y_{1}, y_{2}, \cdots y_{p}$ is gradually reduced in variance.

According to Morrison, the main component should account for approximately $75 \%$ of the total variance. The relevant component is a parameter with an eigenvalue above 1 [34]. Based on this standard, four principle factors to make up the value of urban development quality are obtained. Furthermore, we use the method of efficacy coefficient to guarantee that all final scores are positive as follows:

$$
y^{*}=\frac{y-\min (y)}{\max (y)-\min (y)} \times 0.4+0.6
$$

where $y^{*}$ is the final value of urban development quality; $y$ is the value of urban development quality calculated by PCA; $\min (y)$ is the minimum value of urban development quality calculated by PCA; and $\max (y)$ is the maximum value of urban development quality calculated by PCA.

To illustrate the spatial correlation of urban development quality in an intuitive way, the urban development quality of 285 prefecture-level cities under investigation in 2003 and 2016 are presented in Figure 2. Figure 2 shows two main observations. First, there are clear differences in urban development quality across three regions during 2003-2016, and urban development quality in the eastern, central and western regions is decreasing in turn except for 2013 and 2016. Second, urban development quality in the eastern, central and western regions has maintained an upward trend during 2003-2013, and there has been a slight decline in the three years after reaching the peak in 2013. The above results show that urban development quality in China has been fully upgraded in the past fourteen years, but the downward trend in the last three years should draw our constant attention.

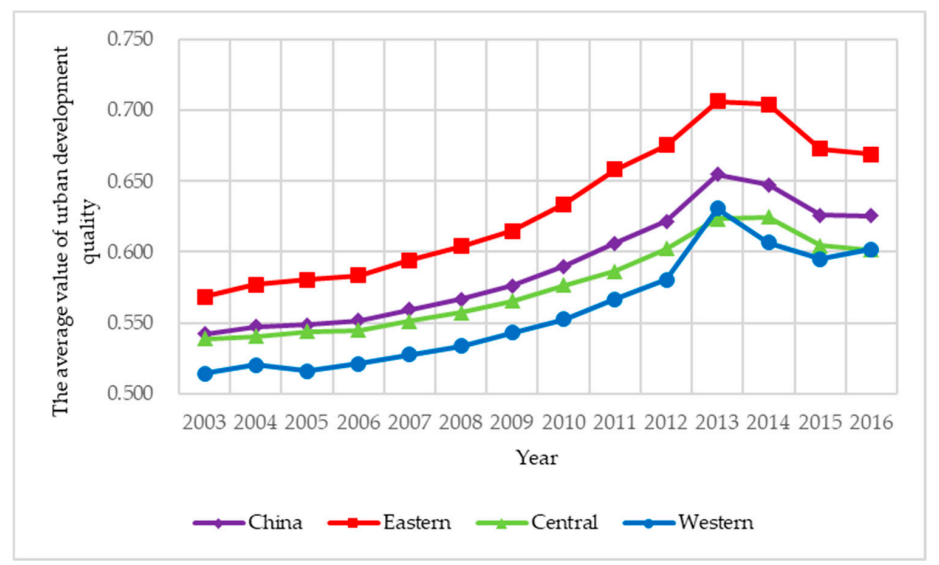

Figure 2. The average value of urban development quality in China during 2003-2016. 


\subsection{Core Explanatory Variables}

Limited to availability, continuity, and comparability of data, sulfur dioxide, and soot and dust are selected as the two main explanatory variables, and based on the difference of action stages, air pollution control is classified into two types: energy saving air pollution control and emission reduction air pollution control. Among them, energy saving air pollution control is the ratio of air pollutants discharged to local GDP, which indicates the cost of economic development; emission reduction air pollution control is the ratio of the removed amount discharged to the produced amount of air pollutants, which indicates the purification degree of air pollutants. To illustrate the spatial correlation of air pollution control in an intuitive way, the four indexes of air pollution control of 285 prefecture-level cities under investigation from 2003 to 2016 are presented in Figures 3-6. Three main observations can be drawn from Figures 3-6. First, the emission intensity of sulfur dioxide has declined rapidly while the emission intensity of smoke and dust has shown wave-like variation. Second, the removal rate of the two air pollutants has been increased rapidly, while the removal rate of smoke and dust is always higher than that of sulfur dioxide. These two pieces of evidence indicate that the control of smoke and dust is better than that of sulfur dioxide. Third, we have investigated that air pollution control in the eastern region is stronger than that in the central region and the western region during 2003-2016 on the whole, implying that local governments in the eastern region generally have deeper consciousness of environment protection and more sufficient supervision of the implement of air pollution control than those in the other two regions.

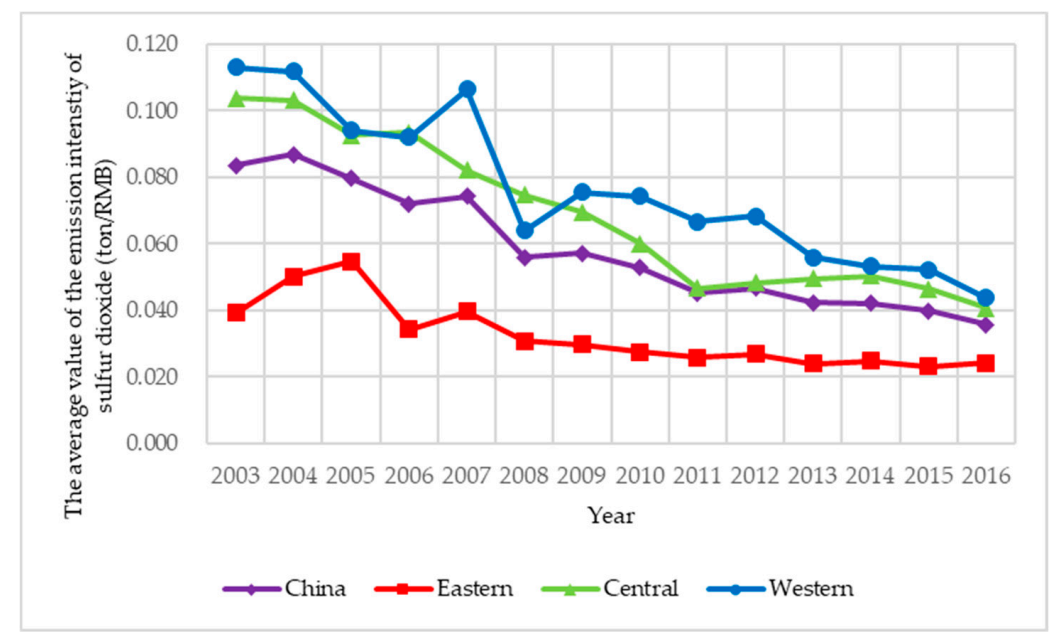

Figure 3. The average value of the emission intensity of sulfur dioxide in China during 2003-2016.

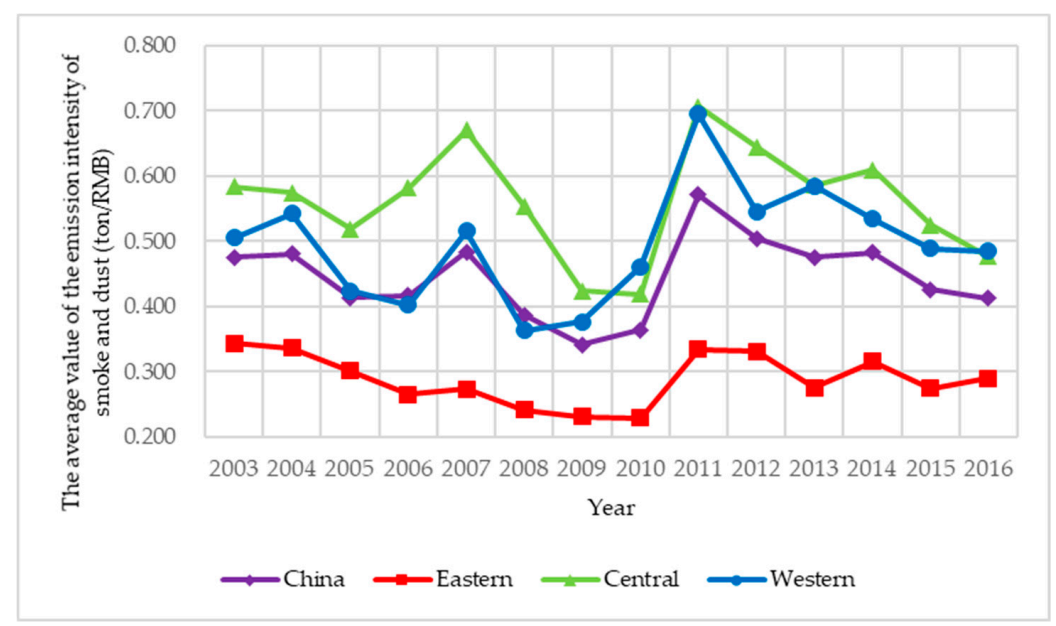

Figure 4. The average value of the emission intensity of smoke and dust in China during 2003-2016. 


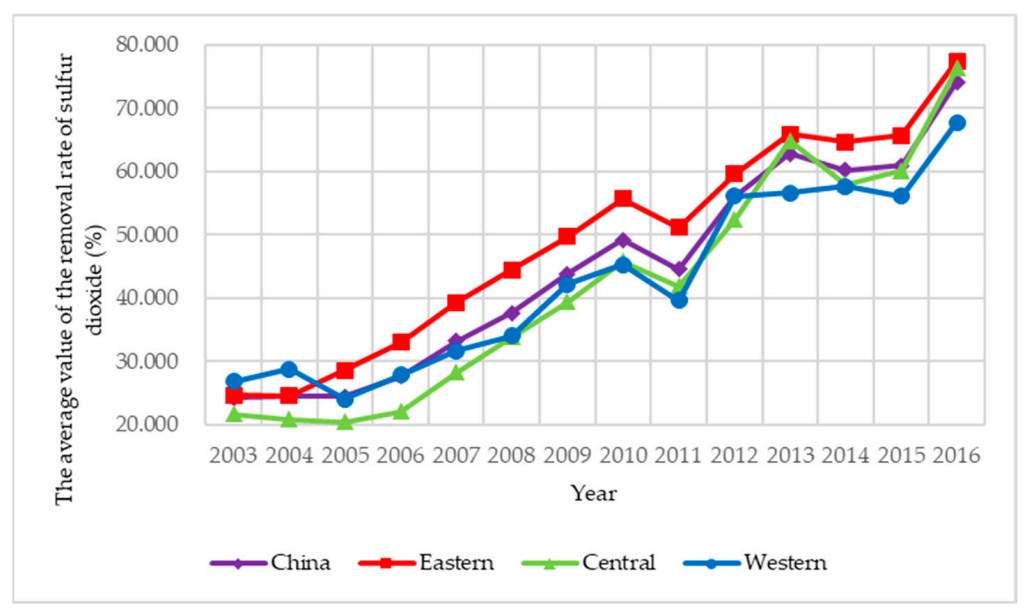

Figure 5. The average value of the removal rate of sulfur dioxide in China during 2003-2016.

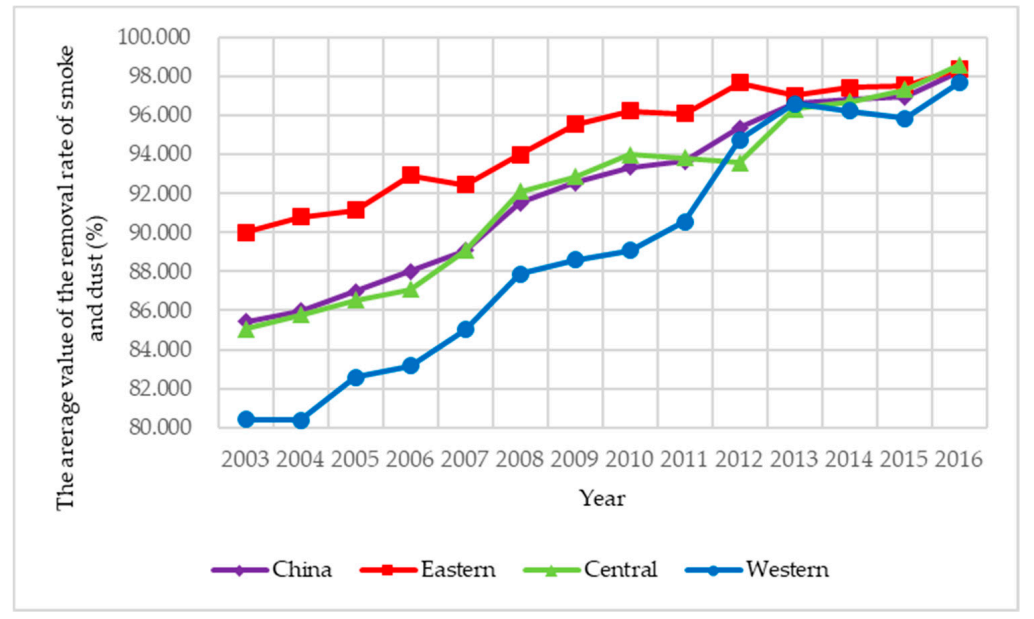

Figure 6. The average value of the removal rate of smoke and dust in China during 2003-2016.

\subsection{Control Variables}

Specific to China, there are also some institutional and economic development factors contributing to the urban development quality in different regions in China. As a result, five control variables are included in the econometric estimation: (1) land finance (LF), i.e., the shares of land leasing revenue in GDP; (2) finance development (FD), i.e., the shares of both deposits and loans in GDP; (3) human capital (HC), i.e., the number of college students per 10,000 people; (4) foreign direct investment (FDI), i.e., the shares of foreign direct investment in GDP, and the annual exchange rate of RMB against the US dollars is used to convert FDI in US dollars to RMB; (5) industrial upgrading (IU), i.e., the shares of the value of the tertiary industries in the value of the secondary industries.

\subsection{Descriptive Statistics and Correlation Coefficients for Regression Variables}

To eliminate the impact of price fluctuations, with the year 2003 as the base period, the economic variables are processed at a constant price. To eliminate the influence of heteroscedasticity, this study has done logarithmic processing to all variables. Table 1 reports the data sources of relevant variables used in this paper. Appendix B also reports the data sources of relevant variables used in this paper. The correlation coefficients presented in Table 2 suggest significant and negative correlation between $\ln E S$ and $\ln U D Q$, indicating the function of energy-saving air pollution control in blocking urban development quality in China; however, there is also a significant and positive correlation between $\ln E R$ and $\ln U D Q$, indicating the role of emission-reduction air pollution control in promoting urban development quality in China. 
Table 1. Descriptive statistics.

\begin{tabular}{|c|c|c|c|c|c|c|c|c|c|c|c|c|}
\hline Variables & Definition & Obs. & Unit & Std. Dev. & Mean & Min & $\begin{array}{c}\text { First } \\
\text { Quartile }\end{array}$ & $\begin{array}{l}\text { Median } \\
\text { Quartile }\end{array}$ & $\begin{array}{c}\text { Third } \\
\text { Quartile }\end{array}$ & $\operatorname{Max}$ & Kurtosis & Skewness \\
\hline $\ln U D O$ & Urban development quality & 3990 & - & 0.185 & -0.536 & -1.047 & -0.647 & -0.570 & -0.464 & 2.257 & 41.015 & 4.005 \\
\hline $\ln E S_{1}$ & The emission intensity of sulfur dioxide per GDP & 3990 & ton/RMB & 1.356 & -3.734 & -14.514 & -4.481 & -3.707 & -2.878 & 1.334 & 3.734 & -0.642 \\
\hline $\ln E S_{2}$ & The emission intensity of smoke and dust per GDP & 3990 & ton/RMB & 1.690 & -1.755 & -12.329 & -2.673 & -1.468 & -0.619 & 1.816 & 2.869 & -1.193 \\
\hline $\ln E R_{1}$ & The removal rate of sulfur dioxide & 3990 & $\%$ & 1.159 & -1.194 & -8.517 & -1.623 & -0.777 & -0.414 & 0.000 & 5.158 & -1.962 \\
\hline $\ln E R_{2}$ & The removal rate of smoke and dust & 3990 & $\%$ & 0.309 & -0.109 & -6.908 & -0.067 & -0.026 & -0.012 & 0.000 & 101.414 & -7.942 \\
\hline $\ln L F$ & The shares of land leasing revenue in GDP & 3990 & $\%$ & 1.536 & -4.928 & -13.119 & -5.743 & -4.408 & -4.122 & -1.419 & 1.924 & -1.099 \\
\hline $\ln F D$ & The shares of both deposits and loans in GDP & 3990 & $\%$ & 0.763 & 1.308 & -4.838 & 0.830 & 1.559 & 1.732 & 4.605 & 10.033 & 0.229 \\
\hline $\ln H C$ & The number of college students per 10,000 people & 3990 & $10^{4}$ persons & 1.174 & 0.940 & -9.210 & 0.376 & 1.179 & 1.653 & 3.177 & 6.36 & -1.696 \\
\hline $\ln F D I$ & The shares of foreign direct investment in GDP & 3990 & $\%$ & 2.954 & -2.922 & -12.512 & -4.682 & -3.693 & -2.449 & 4.605 & 0.311 & 0.879 \\
\hline $\ln I U$ & $\begin{array}{l}\text { The shares of the value of the tertiary industries in } \\
\text { the value of the secondary industries }\end{array}$ & 3990 & $\%$ & 0.517 & -0.172 & -2.361 & -0.484 & -0.177 & 0.137 & 1.621 & 1.377 & -0.073 \\
\hline
\end{tabular}

Table 2. Correlation coefficients for regression variables.

\begin{tabular}{|c|c|c|c|c|c|c|c|c|c|c|}
\hline & $\ln U D Q$ & $\ln E S_{1}$ & $\ln E S_{2}$ & $\ln E R_{1}$ & $\ln E R_{2}$ & $\ln L F$ & $\ln F D$ & $\ln H C$ & $\ln F D I$ & $\ln I U$ \\
\hline $\ln U D Q$ & 1.000 & & & & & & & & & \\
\hline $\ln E S_{1}$ & $-0.250^{* * *}$ & 1.000 & & & & & & & & \\
\hline $\ln E S_{2}$ & $-0.101^{* * *}$ & $0.684^{* * *}$ & 1.000 & & & & & & & \\
\hline $\ln E R_{1}$ & $0.245^{* * *}$ & $0.063^{* * *}$ & $0.078^{* * *}$ & 1.000 & & & & & & \\
\hline $\ln E R_{2}$ & $0.166^{* * *}$ & $0.073^{* * *}$ & $0.356^{* * *}$ & $0.202^{* * *}$ & 1.000 & & & & & \\
\hline $\ln L F$ & $0.151^{* * *}$ & -0.010 & 0.004 & $0.080 * * *$ & $-0.035^{* *}$ & 1.000 & & & & \\
\hline $\ln F D$ & $0.170^{* * *}$ & 0.013 & $0.070^{* * *}$ & $0.169^{* * *}$ & 0.040 * & $0.150 * * *$ & 1.000 & & & \\
\hline $\ln H C$ & $0.321 * * *$ & $-0.039^{* *}$ & 0.011 & $0.114^{* * *}$ & $0.071^{* * *}$ & $0.094^{* * *}$ & 0.024 & 1.000 & & \\
\hline $\ln F D I$ & $-0.027^{*}$ & $0.044^{* * *}$ & -0.014 & -0.017 & $-0.048^{* * *}$ & 0.010 & $0.068^{* * *}$ & $-0.121^{* * *}$ & 1.000 & \\
\hline $\ln I U$ & $0.074^{* * *}$ & $-0.098^{* * *}$ & $-0.11^{* * *}$ & $0.043^{* * *}$ & $-0.091^{* * *}$ & $0.148^{* * *}$ & $0.173^{* * *}$ & $0.094^{* * *}$ & $0.120^{* * *}$ & 1.000 \\
\hline
\end{tabular}

Note: ${ }^{* * *}, * *$ and ${ }^{*}$ denote statistical significance at the $1 \%, 5 \%$, and $10 \%$ significance levels, respectively. 


\section{Analysis and Discussion}

\subsection{Statistical Tests of Unit Root and Granger Causality}

In the test of the unit root, we employed the methods of Levin et al. [36], Im et al. [37], and Maddala and $\mathrm{Wu}$ [38]. With an intercept and linear trend, each of these tests was carried out to include an intercept. As shown in Table 3 below, the unit root tests indicate that all the data series except $\ln U D Q$ and $\ln H C$ are static at a level, however, all the data series become static after the first difference is obtained.

Table 3. Panel unit root test results.

\begin{tabular}{ccccccc}
\hline \multirow{2}{*}{ Variables } & \multicolumn{3}{c}{ Level } & \multicolumn{3}{c}{ First Difference } \\
\cline { 2 - 7 } & $\begin{array}{c}\text { Levin, Liu } \\
\text { and Chu }\end{array}$ & $\begin{array}{c}\text { Im, Pesaran } \\
\text { and Shin }\end{array}$ & ADF & $\begin{array}{c}\text { Levin, Liu } \\
\text { and Chu }\end{array}$ & $\begin{array}{c}\text { Im, Pesaran } \\
\text { and Shin }\end{array}$ & ADF \\
\hline $\ln U D Q$ & $-14.570^{* * *}$ & $-1.097^{* * *}$ & $635.810^{* *}$ & $-13.066^{* * *}$ & $-11.994^{* * *}$ & $1134.650^{* * *}$ \\
$\ln E S_{1}$ & $-24.228^{* * *}$ & $-5.974^{* * *}$ & $780.801^{* * *}$ & $-32.4714^{* * *}$ & $-17.686^{* * *}$ & $1323.270^{* * *}$ \\
$\ln E S_{2}$ & $-58.479^{* * *}$ & $-12.867^{* * *}$ & $923.438^{* * *}$ & $-57.117^{* * *}$ & $-12.728^{* * *}$ & $1594.850^{* * *}$ \\
$\ln E R_{1}$ & $-13.115^{* * *}$ & $-3.601^{* * *}$ & $708.664^{* * *}$ & $-25.195^{* * *}$ & $-15.038^{* * *}$ & $1189.290^{* * *}$ \\
$\ln E R_{2}$ & $-16.644^{* * *}$ & $-4.461^{* * *}$ & $727.268^{* * *}$ & $-26.565^{* * *}$ & $-15.395^{* * *}$ & $1205.780^{* * *}$ \\
$\ln L F$ & $-12.378^{* * *}$ & $-4.888^{* * *}$ & $754.713^{* * *}$ & $-24.878^{* * *}$ & $-17.481^{* * *}$ & $1275.580^{* * *}$ \\
$\ln F D$ & $-9.233^{* * *}$ & $-5.049^{* * *}$ & $699.444^{* * *}$ & $-12.991^{* * *}$ & $-15.432^{* * *}$ & $1203.480^{* * *}$ \\
$\ln H C$ & $0.084^{* * *}$ & $5.779^{* * *}$ & $533.904^{* *}$ & $-5.528^{* * *}$ & $-10.172^{* * *}$ & $1116.980^{* * *}$ \\
$\ln F D I$ & $-40.262^{* * *}$ & $-8.937^{* * *}$ & $844.094^{* * *}$ & $-99.703^{* * *}$ & $-59.857^{* * *}$ & $3159.320^{* * *}$ \\
$\ln I U$ & $-11.750^{* * *}$ & -0.971 & $672.209^{* * *}$ & $-25.091^{* * *}$ & $-17.406^{* * *}$ & $1310.790^{* * *}$ \\
\hline
\end{tabular}

Taking all factors into account, the existence of a unit root at the level and the absence of any at first difference is supported by the results of the unit root test. Additionally, results of both panel Granger causality and bootstrap Granger causality implied that Granger causal relationships run from $\ln E S$ and $\ln E R$ to $\ln U D Q$, rather than bi-directionally. Thus, it is reasonable to further investigate the impact of air pollution control on urban development quality in China.

\subsection{Estimation Results for the Whole Sample}

In the use of spatial Durbin model, spatial dependence is investigated first. The results show that: the global Moran's I index $w_{i j}^{1}$ is 0.202 and $w_{i j}^{2}$ is 0.194 , both inconsistent with the original hypothesis at $1 \%$ significance level, indicating that the spatial econometric model should be selected for statistical verification using the maximum likelihood method. The LR test and the Wald test of spatial Durbin model (SDM) show that the original hypothesis is rejected at the $1 \%$ level of significance, i.e., spatial Durbin model (SDM) cannot degenerate into the spatial lag model (SLM) or spatial error model (SEM). The Hausman test result shows with a $1 \%$ significance level test, the fixed effect model of spatial Durbin model (SDM) should be selected. Further comprehensive analysis of the R_squared, the natural $\log$-likelihood function value $\log \mathrm{L}$, and the joint significance of LR test (space fixed and time fixed) reveal that spatial Durbin model (SDM) is more reasonable under the fixed effect of space-time. Hence, we choose the results of this model for analysis, and the results of the various model tests are shown in Table 4. 
Table 4. The results for the whole sample.

\begin{tabular}{|c|c|c|c|c|c|c|c|c|}
\hline \multirow{2}{*}{$\begin{array}{l}\text { Variables } \\
\text { Constant }\end{array}$} & \multicolumn{4}{|c|}{$w_{i j}^{1}$} & \multicolumn{4}{|c|}{$w_{i j}^{2}$} \\
\hline & $\begin{array}{c}-0.470 * * * \\
(-20.333)\end{array}$ & & & & $\begin{array}{c}-0.464^{* * *} \\
(-19.795)\end{array}$ & & & \\
\hline $\ln E S_{1}$ & $\begin{array}{c}-0.026^{* * *} \\
(-9.378)\end{array}$ & $\begin{array}{c}-0.019 * * * \\
(-5.430)\end{array}$ & $\begin{array}{c}-0.023 * * * \\
(-8.069)\end{array}$ & $\begin{array}{c}-0.009^{* * *} \\
(-2.635)\end{array}$ & $\begin{array}{c}-0.026^{* * *} \\
(-9.619)\end{array}$ & $\begin{array}{c}-0.018^{* * *} \\
(-5.270)\end{array}$ & $\begin{array}{c}-0.024^{* * *} \\
(-8.428)\end{array}$ & $\begin{array}{c}-0.009^{* * *} \\
(-2.622)\end{array}$ \\
\hline $\ln E S_{2}$ & $\begin{array}{l}-0.005^{* *} \\
(-2.177)\end{array}$ & $\begin{array}{c}0.010^{* * *} \\
(4.061)\end{array}$ & $\begin{array}{c}-0.007 * * * \\
(-3.145)\end{array}$ & $\begin{array}{c}0.006^{* * *} \\
(2.694)\end{array}$ & $\begin{array}{l}-0.005^{* *} \\
(-2.325)\end{array}$ & $\begin{array}{c}0.010^{* * *} \\
(3.986)\end{array}$ & $\begin{array}{c}-0.007 * * * \\
(-3.177)\end{array}$ & $\begin{array}{c}0.006^{* * *} \\
(2.721)\end{array}$ \\
\hline $\ln E R_{1}$ & $\begin{array}{c}0.017^{* * * *} \\
(7.224)\end{array}$ & $\begin{array}{c}0.007^{* * *} \\
(2.849)\end{array}$ & $\begin{array}{c}0.014^{* * * *} \\
(5.639)\end{array}$ & $\begin{array}{c}-0.002 \\
(-0.869) \\
\end{array}$ & $\begin{array}{c}0.017^{* * * *} \\
(7.133)\end{array}$ & $\begin{array}{c}0.007^{* * *} \\
(2.709)\end{array}$ & $\begin{array}{c}0.014^{* * *} \\
(5.777)\end{array}$ & $\begin{array}{c}-0.002 \\
(-0.801)\end{array}$ \\
\hline $\ln E R_{2}$ & $\begin{array}{c}0.066^{* * *} \\
(7.372)\end{array}$ & $\begin{array}{c}0.006 \\
(0.743)\end{array}$ & $\begin{array}{c}0.069^{* * *} \\
(7.771)\end{array}$ & $\begin{array}{c}0.003 \\
(0.323)\end{array}$ & $\begin{array}{c}0.066^{* * *} \\
(7.447)\end{array}$ & $\begin{array}{c}0.006 \\
(0.680) \\
\end{array}$ & $\begin{array}{c}0.070 * * * \\
(7.897) \\
\end{array}$ & $\begin{array}{c}0.003 \\
(0.330) \\
\end{array}$ \\
\hline $\ln L F$ & $\begin{array}{c}0.009 * * * \\
(5.383)\end{array}$ & $\begin{array}{c}0.007^{* * *} \\
(4.241)\end{array}$ & $\begin{array}{c}0.008^{* * *} \\
(5.019)\end{array}$ & $\begin{array}{c}0.005^{* * *} \\
(2.987)\end{array}$ & $\begin{array}{c}0.008^{* * *} \\
(5.251)\end{array}$ & $\begin{array}{c}0.007^{* * *} \\
(4.215)\end{array}$ & $\begin{array}{c}0.008^{* * *} \\
(4.902)\end{array}$ & $\begin{array}{c}0.005^{* * *} \\
(2.981)\end{array}$ \\
\hline $\ln F D$ & $\begin{array}{c}0.030 * * * \\
(6.183)\end{array}$ & $\begin{array}{c}0.006 \\
(1.286)\end{array}$ & $\begin{array}{c}0.027 * * * \\
(5.742)\end{array}$ & $\begin{array}{c}0.004 \\
(0.799)\end{array}$ & $\begin{array}{c}0.031^{* * *} \\
(6.539)\end{array}$ & $\begin{array}{c}0.006 \\
(1.314)\end{array}$ & $\begin{array}{c}0.029^{* * *} \\
(6.080)\end{array}$ & $\begin{array}{c}0.004 \\
(0.781)\end{array}$ \\
\hline $\ln H C$ & $\begin{array}{l}0.047^{* * *} \\
(19.616)\end{array}$ & $\begin{array}{c}0.015^{* * *} \\
(4.495)\end{array}$ & $\begin{array}{c}0.047^{* * *} \\
(19.680)\end{array}$ & $\begin{array}{c}0.012^{* * *} \\
(3.656)\end{array}$ & $\begin{array}{l}0.048^{* * *} \\
(20.120)\end{array}$ & $\begin{array}{c}0.016^{* * *} \\
(4.778)\end{array}$ & $\begin{array}{l}0.048^{* * *} \\
(20.103)\end{array}$ & $\begin{array}{c}0.012 * * * \\
(3.745)\end{array}$ \\
\hline $\ln F D I$ & $\begin{array}{c}0.001 \\
(1.001) \\
\end{array}$ & $\begin{array}{c}0.001 \\
(0.567) \\
\end{array}$ & $\begin{array}{c}0.001 \\
(1.238) \\
\end{array}$ & $\begin{array}{c}0.001 \\
(1.041) \\
\end{array}$ & $\begin{array}{c}0.001 \\
(0.650) \\
\end{array}$ & $\begin{array}{c}0.001 \\
(0.618) \\
\end{array}$ & $\begin{array}{c}0.001 \\
(0.898) \\
\end{array}$ & $\begin{array}{c}0.001 \\
(1.025)\end{array}$ \\
\hline $\ln I U$ & $\begin{array}{c}-0.008 \\
(-1.604) \\
\end{array}$ & $\begin{array}{c}0.022 * * * \\
(3.062) \\
\end{array}$ & $\begin{array}{c}-0.008 \\
(-1.542) \\
\end{array}$ & $\begin{array}{c}0.019^{* * *} \\
(2.659) \\
\end{array}$ & $\begin{array}{c}-0.009 \\
(-1.912) \\
\end{array}$ & $\begin{array}{c}0.021^{* * *} \\
(2.994) \\
\end{array}$ & $\begin{array}{l}-0.008^{*} \\
(-1.685) \\
\end{array}$ & $\begin{array}{c}0.019 * * * \\
(2.707) \\
\end{array}$ \\
\hline$w^{*} \mathrm{n} E S_{1}$ & $\begin{array}{c}-0.013^{* * *} \\
(-3.277)\end{array}$ & $\begin{array}{c}-0.034^{* * *} \\
(-7.051)\end{array}$ & $\begin{array}{c}-0.004 \\
(-0.966)\end{array}$ & $\begin{array}{c}-0.003 \\
(-0.614) \\
\end{array}$ & $\begin{array}{c}-0.011^{* * *} \\
(-2.936)\end{array}$ & $\begin{array}{c}-0.035^{* * *} \\
(-7.354)\end{array}$ & $\begin{array}{c}-0.004 \\
(-0.943)\end{array}$ & $\begin{array}{c}-0.005 \\
(-0.922)\end{array}$ \\
\hline$w^{*} \ln E S_{2}$ & $\begin{array}{c}0.010 * * * \\
(3.224)\end{array}$ & $\begin{array}{c}0.018^{* * *} \\
(4.634)\end{array}$ & $\begin{array}{c}0.004 \\
(1.166)\end{array}$ & $\begin{array}{l}0.007 * \\
(1.912)\end{array}$ & $\begin{array}{c}0.011^{* * *} \\
(3.510)\end{array}$ & $\begin{array}{c}0.018^{* * *} \\
(4.676)\end{array}$ & $\begin{array}{c}0.006 \\
(1.624)\end{array}$ & $\begin{array}{l}0.008^{* *} \\
(2.121)\end{array}$ \\
\hline$w^{*} \ln E R_{1}$ & $\begin{array}{c}0.017^{* * *} \\
(5.311)\end{array}$ & $\begin{array}{c}0.019^{* * *} \\
(6.042)\end{array}$ & $\begin{array}{c}0.010^{* * *} \\
(2.701)\end{array}$ & $\begin{array}{c}-0.001 \\
(-0.375)\end{array}$ & $\begin{array}{c}0.018^{* * *} \\
(5.448)\end{array}$ & $\begin{array}{c}0.019^{* * *} \\
(5.741)\end{array}$ & $\begin{array}{c}0.012 * * * \\
(3.053)\end{array}$ & $\begin{array}{c}-0.002 \\
(-0.635)\end{array}$ \\
\hline$w^{*} \ln E R_{2}$ & $\begin{array}{c}0.001 \\
(0.069)\end{array}$ & $\begin{array}{c}-0.001 \\
(-0.083)\end{array}$ & $\begin{array}{c}0.012 \\
(0.863)\end{array}$ & $\begin{array}{c}-0.005 \\
(-0.398)\end{array}$ & $\begin{array}{c}0.001 \\
(0.071)\end{array}$ & $\begin{array}{c}-0.004 \\
(-0.285)\end{array}$ & $\begin{array}{c}0.013 \\
(0.875)\end{array}$ & $\begin{array}{l}-0.006 \\
(-0.45)\end{array}$ \\
\hline
\end{tabular}


Table 4. Cont.

\begin{tabular}{|c|c|c|c|c|c|c|c|c|}
\hline \multirow{2}{*}{$\begin{array}{c}\text { Variables } \\
w^{*} \ln L F\end{array}$} & \multicolumn{4}{|c|}{$w_{i j}^{1}$} & \multicolumn{4}{|c|}{$w_{i j}^{2}$} \\
\hline & $\begin{array}{c}0.003 \\
(1.051) \\
\end{array}$ & $\begin{array}{c}0.002 \\
(0.828) \\
\end{array}$ & $\begin{array}{c}0.001 \\
(0.544) \\
\end{array}$ & $\begin{array}{c}-0.003 \\
(-1.211) \\
\end{array}$ & $\begin{array}{c}0.002 \\
(0.907) \\
\end{array}$ & $\begin{array}{l}0.002 \\
(0.96) \\
\end{array}$ & $\begin{array}{c}0.001 \\
(0.535) \\
\end{array}$ & $\begin{array}{c}-0.002 \\
(-0.912) \\
\end{array}$ \\
\hline$w^{*} \ln F D$ & $\begin{array}{c}-0.016 \text { *** } \\
(-2.897)\end{array}$ & $\begin{array}{c}0.008 \\
(1.500)\end{array}$ & $\begin{array}{c}-0.023 * * * \\
(-3.906)\end{array}$ & $\begin{array}{c}-0.004 \\
(-0.667)\end{array}$ & $\begin{array}{l}-0.464^{* * *} \\
(-19.795)\end{array}$ & $\begin{array}{c}0.000 \\
(0.000)\end{array}$ & $\begin{array}{c}0.000 \\
(0.000)\end{array}$ & $\begin{array}{c}0.000 \\
(0.000)\end{array}$ \\
\hline$w^{*} \ln H C$ & $\begin{array}{c}-0.018^{* * *} \\
(-5.743)\end{array}$ & $\begin{array}{l}-0.008 \text { ** } \\
(-2.017)\end{array}$ & $\begin{array}{c}-0.015^{* * *} \\
(-4.693)\end{array}$ & $\begin{array}{c}-0.013 * * * \\
(-3.329)\end{array}$ & $\begin{array}{c}-0.026^{* * *} \\
(-9.619)\end{array}$ & $\begin{array}{c}-0.018^{* * *} \\
(-5.270)\end{array}$ & $\begin{array}{c}-0.024^{* * *} \\
(-8.428)\end{array}$ & $\begin{array}{c}-0.009 \text { *** } \\
(-2.622)\end{array}$ \\
\hline$w^{*} \ln F D I$ & $\begin{array}{c}0.000 \\
(0.030)\end{array}$ & $\begin{array}{c}-0.002 \\
(-1.583)\end{array}$ & $\begin{array}{c}0.001 \\
(0.555)\end{array}$ & $\begin{array}{c}-0.001 \\
(-0.804)\end{array}$ & $\begin{array}{l}-0.005^{* *} \\
(-2.325)\end{array}$ & $\begin{array}{c}0.010 * * * \\
(3.986)\end{array}$ & $\begin{array}{c}-0.007^{* * *} \\
(-3.177)\end{array}$ & $\begin{array}{c}0.006^{* * *} \\
(2.721)\end{array}$ \\
\hline$w^{*} \ln I U$ & $\begin{array}{c}0.022 * * * \\
(3.086) \\
\end{array}$ & $\begin{array}{c}0.001 \\
(0.109) \\
\end{array}$ & $\begin{array}{c}0.023 * * * \\
(3.190) \\
\end{array}$ & $\begin{array}{c}-0.003 \\
(-0.303) \\
\end{array}$ & $\begin{array}{c}0.017 * * * \\
(7.133) \\
\end{array}$ & $\begin{array}{c}0.007^{* * *} \\
(2.709) \\
\end{array}$ & $\begin{array}{c}0.014 * * * \\
(5.777) \\
\end{array}$ & $\begin{array}{c}-0.002 \\
(-0.801) \\
\end{array}$ \\
\hline$\rho$ & $\begin{array}{c}0.257^{* * *} \\
(15.552)\end{array}$ & $\begin{array}{c}0.208^{* * *} \\
(12.261)\end{array}$ & $\begin{array}{c}0.234^{* * *} \\
(13.927)\end{array}$ & $\begin{array}{c}0.126^{* * *} \\
(7.105)\end{array}$ & $\begin{array}{c}0.066^{* * *} \\
(7.447)\end{array}$ & $\begin{array}{c}0.006 \\
(0.680)\end{array}$ & $\begin{array}{c}0.070^{* * *} \\
(7.897)\end{array}$ & $\begin{array}{c}0.003 \\
(0.330) \\
\end{array}$ \\
\hline Space-fixed & No & Yes & No & Yes & No & Yes & No & Yes \\
\hline Time-fixed & No & No & Yes & Yes & No & No & Yes & Yes \\
\hline R-squared & 0.330 & 0.567 & 0.345 & 0.594 & 0.330 & 0.567 & 0.345 & 0.594 \\
\hline Log-likelihood & 1825.446 & 2708.772 & 1876.033 & 2860.128 & 1826.725 & 2709.785 & 1877.126 & 2860.577 \\
\hline Moran's I & \multicolumn{4}{|c|}{$0.202^{* * *}$} & \multicolumn{4}{|c|}{$0.194^{* * *}$} \\
\hline LR_joint_space fixed & \multicolumn{4}{|c|}{$1112.135^{* * *}$} & \multicolumn{4}{|c|}{$1126.444^{* * *}$} \\
\hline LR_joint_time fixed & \multicolumn{4}{|c|}{$277.543^{* * *}$} & \multicolumn{4}{|c|}{$277.030^{* * *}$} \\
\hline Wald_spatial_lag & \multirow{2}{*}{\multicolumn{4}{|c|}{$\begin{array}{l}17.416^{* *} \\
18.673^{* *}\end{array}$}} & \multicolumn{4}{|c|}{$18.580^{* *}$} \\
\hline LR_spatial_lag & & & & & \multicolumn{4}{|c|}{$20.004^{* *}$} \\
\hline Wald_spatial_error & & & & & \multicolumn{4}{|c|}{$18.035^{* *}$} \\
\hline LR_spatial_error & \multicolumn{4}{|c|}{$17.810^{* *}$} & & & & \\
\hline Hauman test & \multicolumn{4}{|c|}{$1066.035^{* * *}$} & \multicolumn{4}{|c|}{$1208.316^{* * *}$} \\
\hline obs & 3990 & 3990 & 3990 & 3990 & 3990 & 3990 & 3990 & 3990 \\
\hline
\end{tabular}


As can be seen in Table 4, the coefficients of four air pollution control indexes are consistent, indicating that the specification of the spatial weights matrices has no effect on the estimation results. Estimation results of the spatial Durbin model (SDM) in Table 4 show that $\ln E S_{1}$ has a significantly negative correlation with $\ln U D Q$, while $\ln E S_{2}$ has a significantly positive correlation with $\ln U D Q$. As discussed previously, the air pollution control during the sample period is mainly focused on smoke and dust emissions, while emissions of sulfur dioxide during the sample period are basically increasing, which is the evidence for the relationship between energy-saving air pollution control and urban development quality. However, the relationship between emission-reduction air pollution control and urban development quality is not significantly associated with $\ln U D Q$, indicating that the effect of air pollution control on urban development quality is not satisfactory. Furthermore, the coefficients of $\ln L F$, $\ln H C$ and $\ln I U$ also have a significantly positive association with $\ln U D Q$, indicating the enhancement of land finance, human capital and industrial upgrading on urban development. Moreover, the coefficients of $\ln F D$ and $\ln F D I$ are also not significantly associated with $\ln U D Q$, indicating that those two factors are weak in promoting urban development quality. As for the coefficients of the spatial item, only $w^{*} \ln E S_{2}$ has significantly positive correlation with $\ln U D Q$ and $w^{*} \ln H C$ is has a significantly negative correlation with it, while others are not significantly associated with $\ln U D Q$, showing the importance of air pollution control in sulfur dioxide. In addition, the spatial coefficients $(\rho)$ are also highly significant, which is a strong evidence of spatial dependence of urban development quality.

By introducing spatial effects into the traditional data model, the impact of air pollution control on urban development quality is no longer reflected only in the explanatory variables' coefficient; instead, the spatial effect allows the impact to be disaggregated into direct and indirect effects.

As is shown in Table 5, the coefficients of direct effect and indirect effect are nearly consistent with the corresponding value in Table 4, indicating that the spatial feedback effects among different cities are negligible. The total effect of $\ln E S_{1}$ is significantly negatively correlated with $\ln U D Q$, while $\ln E S_{2}$ is significantly positively correlated with $\ln U D Q$ and the other variables are not significant statistically, implying the importance of energy-saving air pollution control on urban development quality.

Table 5. The direct, indirect and total effects of the whole sample.

\begin{tabular}{|c|c|c|c|c|c|c|}
\hline \multirow{2}{*}{ Variables } & \multicolumn{3}{|c|}{$w_{i j}^{1}$} & \multicolumn{3}{|c|}{$w_{i j}^{2}$} \\
\hline & Direct Effect & Indirect Effect & Total Effect & Direct Effect & Indirect Effect & Total Effect \\
\hline $\ln E S_{1}$ & $\begin{array}{c}-0.010^{* * *} \\
(-2.768)\end{array}$ & $\begin{array}{c}-0.005 \\
(-0.755)\end{array}$ & $\begin{array}{l}-0.014^{* *} \\
(-2.003)\end{array}$ & $\begin{array}{c}-0.009^{* * *} \\
(-2.815)\end{array}$ & $\begin{array}{c}-0.007 \\
(-1.108)\end{array}$ & $\begin{array}{l}-0.016^{* *} \\
(-2.324)\end{array}$ \\
\hline $\ln E S_{2}$ & $\begin{array}{c}0.007^{* * *} \\
(2.800)\end{array}$ & $\begin{array}{c}0.009^{* *} \\
(2.037)\end{array}$ & $\begin{array}{c}0.016 \text { *** } \\
(3.042)\end{array}$ & $\begin{array}{c}0.007^{* * *} \\
(2.824)\end{array}$ & $\begin{array}{c}0.010^{* *} \\
(2.238)\end{array}$ & $\begin{array}{c}0.017^{* * *} \\
(3.298)\end{array}$ \\
\hline $\ln E R_{1}$ & $\begin{array}{c}-0.002 \\
(-0.934)\end{array}$ & $\begin{array}{c}-0.002 \\
(-0.422)\end{array}$ & $\begin{array}{c}-0.004 \\
(-0.815)\end{array}$ & $\begin{array}{c}-0.002 \\
(-0.812)\end{array}$ & $\begin{array}{c}-0.003 \\
(-0.744)\end{array}$ & $\begin{array}{c}-0.005 \\
(-1.020)\end{array}$ \\
\hline $\ln E R_{2}$ & $\begin{array}{c}0.002 \\
(0.271)\end{array}$ & $\begin{array}{c}-0.006 \\
(-0.388)\end{array}$ & $\begin{array}{c}-0.003 \\
(-0.185)\end{array}$ & $\begin{array}{c}0.002 \\
(0.282)\end{array}$ & $\begin{array}{c}-0.007 \\
(-0.480)\end{array}$ & $\begin{array}{c}-0.005 \\
(-0.259)\end{array}$ \\
\hline $\ln L F$ & $\begin{array}{c}0.005^{* * *} \\
(2.966)\end{array}$ & $\begin{array}{c}-0.003 \\
(-0.915)\end{array}$ & $\begin{array}{c}0.002 \\
(0.647)\end{array}$ & $\begin{array}{c}0.005^{* * *} \\
(2.876)\end{array}$ & $\begin{array}{c}-0.002 \\
(-0.655)\end{array}$ & $\begin{array}{c}0.003 \\
(0.912)\end{array}$ \\
\hline $\ln F D$ & $\begin{array}{c}0.004 \\
(0.816)\end{array}$ & $\begin{array}{c}-0.004 \\
(-0.640)\end{array}$ & $\begin{array}{c}0.000 \\
(-0.022)\end{array}$ & $\begin{array}{c}0.004 \\
(0.802)\end{array}$ & $\begin{array}{c}-0.003 \\
(-0.548)\end{array}$ & $\begin{array}{c}0.000 \\
(0.088)\end{array}$ \\
\hline $\ln H C$ & $\begin{array}{c}0.011^{* * *} \\
(3.420)\end{array}$ & $\begin{array}{c}-0.013^{* * *} \\
(-3.209)\end{array}$ & $\begin{array}{c}-0.001 \\
(-0.382)\end{array}$ & $\begin{array}{c}0.011^{* * *} \\
(3.596)\end{array}$ & $\begin{array}{c}-0.013^{* * *} \\
(-3.282)\end{array}$ & $\begin{array}{c}-0.002 \\
(-0.439)\end{array}$ \\
\hline $\ln F D I$ & $\begin{array}{c}0.001 \\
(0.980) \\
\end{array}$ & $\begin{array}{c}-0.001 \\
(-0.706) \\
\end{array}$ & $\begin{array}{c}0.000 \\
(-0.064) \\
\end{array}$ & $\begin{array}{c}0.001 \\
(1.028) \\
\end{array}$ & $\begin{array}{c}-0.001 \\
(-0.658) \\
\end{array}$ & $\begin{array}{c}0.000 \\
(-0.012) \\
\end{array}$ \\
\hline $\ln I U$ & $\begin{array}{c}0.019 * * * \\
(2.652)\end{array}$ & $\begin{array}{c}0.000 \\
(0.003)\end{array}$ & $\begin{array}{c}0.019 \\
(1.363)\end{array}$ & $\begin{array}{c}0.019 * * * \\
(2.700)\end{array}$ & $\begin{array}{c}-0.001 \\
(-0.053)\end{array}$ & $\begin{array}{c}0.019 \\
(1.332)\end{array}$ \\
\hline
\end{tabular}

Notes: The t-statistics are given in the parentheses; ${ }^{* * *}$ and ${ }^{* *}$ denote statistical significance at the $1 \%$ and $5 \%$ significance levels, respectively.

\subsection{Estimation Results for the Sub-Regional Sample}

China is a big country with vast territory. Therefore, the impact of air pollution control on urban development quality in different regions varies greatly. To take full account of the differences in urban development quality across regions, the regression is re-estimated using the sub-samples of three 
geographical regions (i.e., eastern, central and western) proposed by the National Bureau of Statistics (NBS). The prefecture-level cities in each region are listed in Appendix A. The estimation results for regression in these three regions are reported in Table 6.

Table 6. The results of the sub-regional sample.

\begin{tabular}{|c|c|c|c|c|c|c|}
\hline \multirow{2}{*}{ Variables } & \multicolumn{2}{|c|}{ Eastern Region } & \multicolumn{2}{|c|}{ Central Region } & \multicolumn{2}{|c|}{ Western Region } \\
\hline & $w_{i j}^{1}$ & $w_{i j}^{2}$ & $w_{i j}^{1}$ & $w_{i j}^{2}$ & $w_{i j}^{1}$ & $w_{i j}^{2}$ \\
\hline $\ln E S_{1}$ & $\begin{array}{c}-0.016^{* * *} \\
(-3.088) \\
\end{array}$ & $\begin{array}{c}-0.016^{* * *} \\
(-3.096) \\
\end{array}$ & $\begin{array}{c}-0.006 \\
(-0.575) \\
\end{array}$ & $\begin{array}{c}-0.005 \\
(-0.492) \\
\end{array}$ & $\begin{array}{c}-0.003 \\
(-0.731) \\
\end{array}$ & $\begin{array}{c}-0.003 \\
(-0.744) \\
\end{array}$ \\
\hline $\ln E S_{2}$ & $\begin{array}{l}0.007^{*} \\
(1.755)\end{array}$ & $\begin{array}{l}0.007^{*} \\
(1.799)\end{array}$ & $\begin{array}{c}0.008 \\
(1.413)\end{array}$ & $\begin{array}{c}0.009 \\
(1.478)\end{array}$ & $\begin{array}{c}0.001 \\
(0.469)\end{array}$ & $\begin{array}{c}0.001 \\
(0.433)\end{array}$ \\
\hline $\ln E R_{1}$ & $\begin{array}{l}-0.010^{*} \\
(-1.779)\end{array}$ & $\begin{array}{l}-0.010 * \\
(-1.756)\end{array}$ & $\begin{array}{c}-0.002 \\
(-0.422)\end{array}$ & $\begin{array}{c}-0.002 \\
(-0.381)\end{array}$ & $\begin{array}{c}0.002 \\
(0.931)\end{array}$ & $\begin{array}{c}0.002 \\
(0.912)\end{array}$ \\
\hline $\ln E R_{2}$ & $\begin{array}{c}0.042^{* *} \\
(2.071)\end{array}$ & $\begin{array}{c}0.042 * * \\
(2.065)\end{array}$ & $\begin{array}{c}0.018 \\
(0.822)\end{array}$ & $\begin{array}{c}0.017 \\
(0.769)\end{array}$ & $\begin{array}{c}-0.007 \\
(-0.962)\end{array}$ & $\begin{array}{c}-0.007 \\
(-0.946)\end{array}$ \\
\hline $\ln L F$ & $\begin{array}{c}0.006^{* *} \\
(2.104)\end{array}$ & $\begin{array}{c}0.006^{* *} \\
(2.081)\end{array}$ & $\begin{array}{c}0.003 \\
(1.040)\end{array}$ & $\begin{array}{c}0.003 \\
(1.037)\end{array}$ & $\begin{array}{l}0.004^{* *} \\
(2.296)\end{array}$ & $\begin{array}{c}0.004^{* *} \\
(2.264)\end{array}$ \\
\hline $\ln F D$ & $\begin{array}{c}-0.006 \\
(-0.712)\end{array}$ & $\begin{array}{c}-0.006 \\
(-0.691)\end{array}$ & $\begin{array}{c}0.009 \\
(0.798)\end{array}$ & $\begin{array}{c}0.007 \\
(0.654)\end{array}$ & $\begin{array}{c}-0.004 \\
(-0.687)\end{array}$ & $\begin{array}{c}-0.004 \\
(-0.583)\end{array}$ \\
\hline $\ln H C$ & $\begin{array}{c}0.041^{* * *} \\
(5.469)\end{array}$ & $\begin{array}{c}0.041^{* * *} \\
(5.465)\end{array}$ & $\begin{array}{l}0.021^{* *} \\
(2.560)\end{array}$ & $\begin{array}{l}0.021^{* *} \\
(2.506)\end{array}$ & $\begin{array}{c}-0.001 \\
(-0.329)\end{array}$ & $\begin{array}{c}-0.001 \\
(-0.322)\end{array}$ \\
\hline $\ln F D I$ & $\begin{array}{c}0.007^{* *} \\
(2.192)\end{array}$ & $\begin{array}{c}0.007 \text { ** } \\
(2.094)\end{array}$ & $\begin{array}{c}-0.002 \\
(-0.679)\end{array}$ & $\begin{array}{c}-0.001 \\
(-0.594)\end{array}$ & $\begin{array}{c}0.001 \\
(0.973)\end{array}$ & $\begin{array}{c}0.001 \\
(0.968)\end{array}$ \\
\hline $\ln I U$ & $\begin{array}{l}0.036^{* *} \\
(2.257)\end{array}$ & $\begin{array}{c}0.038^{* *} \\
(2.338)\end{array}$ & $\begin{array}{c}0.013 \\
(0.862)\end{array}$ & $\begin{array}{c}0.013 \\
(0.912)\end{array}$ & $\begin{array}{c}0.007 \\
(0.895)\end{array}$ & $\begin{array}{c}0.007 \\
(0.955)\end{array}$ \\
\hline$w^{*} \ln E S_{1}$ & $\begin{array}{c}-0.009 \\
(-1.077)\end{array}$ & $\begin{array}{c}-0.011 \\
(-1.311)\end{array}$ & $\begin{array}{c}0.021 \\
(1.401)\end{array}$ & $\begin{array}{c}0.012 \\
(0.798)\end{array}$ & $\begin{array}{c}0.005 \\
(0.753)\end{array}$ & $\begin{array}{c}0.006 \\
(0.947)\end{array}$ \\
\hline$w^{*} \ln E S_{2}$ & $\begin{array}{c}0.014^{* *} \\
(2.241)\end{array}$ & $\begin{array}{l}0.013 * * \\
(2.181)\end{array}$ & $\begin{array}{c}0.002 \\
(0.181)\end{array}$ & $\begin{array}{c}0.005 \\
(0.600)\end{array}$ & $\begin{array}{c}0.004 \\
(0.972)\end{array}$ & $\begin{array}{l}0.004 \\
(0.94)\end{array}$ \\
\hline$w^{*} \ln E R_{1}$ & $\begin{array}{c}-0.004 \\
(-0.460)\end{array}$ & $\begin{array}{l}-0.002 \\
(-0.28)\end{array}$ & $\begin{array}{c}-0.008 \\
(-1.186)\end{array}$ & $\begin{array}{c}-0.009 \\
(-1.325)\end{array}$ & $\begin{array}{l}0.007^{*} \\
(1.947)\end{array}$ & $\begin{array}{l}0.007^{*} \\
(1.702)\end{array}$ \\
\hline$w^{*} \ln E R_{2}$ & $\begin{array}{c}-0.042 \\
(-1.085)\end{array}$ & $\begin{array}{c}-0.042 \\
(-0.998)\end{array}$ & $\begin{array}{l}0.059^{* *} \\
(2.118)\end{array}$ & $\begin{array}{l}0.057^{*} \\
(1.798)\end{array}$ & $\begin{array}{l}-0.014 \\
(-1.09)\end{array}$ & $\begin{array}{c}-0.009 \\
(-0.753)\end{array}$ \\
\hline$w^{*} \ln L F$ & $\begin{array}{c}-0.002 \\
(-0.437)\end{array}$ & $\begin{array}{c}-0.001 \\
(-0.122)\end{array}$ & $\begin{array}{c}-0.005 \\
(-0.973)\end{array}$ & $\begin{array}{c}-0.004 \\
(-0.746)\end{array}$ & $\begin{array}{c}0.001 \\
(0.416)\end{array}$ & $\begin{array}{c}0.001 \\
(0.310)\end{array}$ \\
\hline$w^{*} \ln F D$ & $\begin{array}{c}-0.008 \\
(-0.608)\end{array}$ & $\begin{array}{c}-0.006 \\
(-0.489)\end{array}$ & $\begin{array}{c}-0.008 \\
(-0.444)\end{array}$ & $\begin{array}{c}-0.002 \\
(-0.105)\end{array}$ & $\begin{array}{c}0.003 \\
(0.394)\end{array}$ & $\begin{array}{c}0.002 \\
(0.271)\end{array}$ \\
\hline$w^{*} \ln H C$ & $\begin{array}{c}-0.016 \\
(-1.549)\end{array}$ & $\begin{array}{c}-0.017 \\
(-1.550)\end{array}$ & $\begin{array}{c}-0.036^{* * *} \\
(-3.048)\end{array}$ & $\begin{array}{c}-0.034 * * * \\
(-2.997)\end{array}$ & $\begin{array}{c}0.004 \\
(0.946)\end{array}$ & $\begin{array}{c}0.004 \\
(0.874)\end{array}$ \\
\hline$w^{*} \ln F D I$ & $\begin{array}{l}-0.006^{*} \\
(-1.645) \\
\end{array}$ & $\begin{array}{c}-0.006 \\
(-1.513) \\
\end{array}$ & $\begin{array}{c}-0.003 \\
(-1.056) \\
\end{array}$ & $\begin{array}{c}-0.004 \\
(-1.056) \\
\end{array}$ & $\begin{array}{c}0.000 \\
(0.223)\end{array}$ & $\begin{array}{c}0.001 \\
(0.391)\end{array}$ \\
\hline$w^{*} \ln I U$ & $\begin{array}{c}-0.036 \\
(-1.423)\end{array}$ & $\begin{array}{c}-0.036 \\
(-1.459)\end{array}$ & $\begin{array}{c}-0.032 \\
(-1.437)\end{array}$ & $\begin{array}{c}-0.032 \\
(-1.471)\end{array}$ & $\begin{array}{c}-0.006 \\
(-0.588)\end{array}$ & $\begin{array}{c}-0.001 \\
(-0.125)\end{array}$ \\
\hline$\rho$ & $\begin{array}{c}0.200 * * * \\
(7.215) \\
\end{array}$ & $\begin{array}{c}0.209^{* * *} \\
(7.565) \\
\end{array}$ & $\begin{array}{c}0.026 \\
(0.875)\end{array}$ & $\begin{array}{c}0.032 \\
(1.058)\end{array}$ & $\begin{array}{l}0.058^{*} \\
(1.869)\end{array}$ & $\begin{array}{l}0.054 * \\
(1.728)\end{array}$ \\
\hline Space-fixed & Yes & Yes & Yes & Yes & Yes & Yes \\
\hline Time-fixed & Yes & Yes & Yes & Yes & Yes & Yes \\
\hline R-squared & 0.597 & 0.597 & 0.489 & 0.488 & 0.694 & 0.694 \\
\hline Log-likelihood & 974.957 & 974.424 & 820.675 & 820.077 & 1297.524 & 1296.937 \\
\hline Moran's I & $0.247 * * *$ & $0.240^{* * *}$ & $0.076^{* * *}$ & $0.066^{* * *}$ & $0.127^{* * *}$ & $0.124 * * *$ \\
\hline LR_joint_space fixed & $282.001 * * *$ & $284.884 * * *$ & $354.704 * * *$ & $355.597 * * *$ & $464.838 * * *$ & $465.884 * * *$ \\
\hline LR_joint_time fixed & $111.213^{* * *}$ & $111.311^{* * *}$ & $93.872 * * *$ & $94.043^{* * *}$ & $162.776^{* * *}$ & $157.463^{* * *}$ \\
\hline Wald_spatial_lag & 14.646 & 13.997 & $19.022 * *$ & $17.887^{* *}$ & 9.606 & 8.810 \\
\hline LR_spatial_lag & 15.740 * & $15.059 *$ & $20.461 * *$ & $19.344^{* *}$ & 10.514 & 9.967 \\
\hline Wald_spatial_error & 12.960 & 12.702 & 18.940 ** & $17.812 * *$ & 9.883 & 9.060 \\
\hline LR_spatial_error & 14.160 & 13.829 & $20.394 * *$ & $19.311 * *$ & 10.771 & 9.880 \\
\hline $\begin{array}{l}\text { Hauman test } \\
\text { obs }\end{array}$ & $\begin{array}{c}208.849^{* * *} \\
1414\end{array}$ & $\begin{array}{c}153.087^{* * *} \\
1414\end{array}$ & $\begin{array}{c}26.768 * \\
1400\end{array}$ & $\begin{array}{c}61.305^{* * *} \\
1400\end{array}$ & $\begin{array}{c}336.7211^{* * *} \\
1176\end{array}$ & $\begin{array}{c}378.552 * * * \\
1176\end{array}$ \\
\hline
\end{tabular}

Notes: The t-statistics are given in the parentheses; ${ }^{* * *}, * *$ and ${ }^{*}$ denote statistical significance at the $1 \%, 5 \%$, and $10 \%$ significance levels, respectively.

Generally speaking, the results of three different regions are inconsistent with the results of the whole sample, which means the spatial heterogeneity of different regions is significant. Similar to the estimation results using the whole sample, the intensity of sulfur dioxide fails to play the expected role in promoting the increase of urban development quality, as the coefficients turn out to be negative (although not significant in the central region and western region). Furthermore, the intensity of smoke and dust are the core factor to improve the urban development quality, as the coefficients turn out to be 
positive (although not significant in the central region and western region). Moreover, the coefficients of $\ln E R_{1}$ and $\ln E R_{2}$ are significantly negatively and positively correlated with $\ln U D Q$ respectively in the eastern region, indicating that the difference of air pollution control on urban development quality is more prominent, in the eastern region which is the core of economic and industrial activities. Moreover, the coefficients of control variables in eastern region are similar to the estimation results of the whole sample except $\ln F D I$, which means that FDI has played an important role in promoting the urban development quality in the eastern region. It is noteworthy that most of the coefficients are not significant in the central and western regions. As for the central region, only the coefficient of $\ln H C$ is significantly positively associated with $\ln U D Q$, implying the importance of human capital in promoting the quality of urban development. As for the western region, only the coefficient of $\ln L F$ is significantly positively correlated with $\ln U D Q$, implying the importance of land finance in promoting the quality of urban development. In addition, the spatial coefficients $(\rho)$ are also highly significant in the eastern and western regions, but not significant in the central region, indicating the differentiation of spatial dependence in different regions.

Table 7 reports the direct, indirect and total effects of the eastern region. As is shown in Table 7, the coefficients of direct effect and indirect effect are nearly consistent with the corresponding coefficient in Table 6, indicating that the spatial feedback effects among eastern cities are also negligible. The total effect of $\ln E S_{1}$ has a significantly negative correlation with $\ln U D Q$, while $\ln E S_{2}$ and $\ln H C$ have a significantly positive correlation with $\ln U D Q$, while other variables are not statistically significant, implying the importance of energy-saving air pollution control and human capital on urban development quality in the eastern region.

Table 7. The direct, indirect and total effects of the eastern region.

\begin{tabular}{|c|c|c|c|c|c|c|}
\hline \multirow{2}{*}{ Variables } & \multicolumn{3}{|c|}{$w_{i j}^{1}$} & \multicolumn{3}{|c|}{$w_{i j}^{2}$} \\
\hline & Direct Effect & Indirect Effect & Total Effect & Direct Effect & Indirect Effect & Total Effect \\
\hline $\ln E S_{1}$ & $\begin{array}{c}-0.017^{* * *} \\
(-3.395)\end{array}$ & $\begin{array}{c}-0.014 \\
(-1.447)\end{array}$ & $\begin{array}{c}-0.031 * * * \\
(-2.730)\end{array}$ & $\begin{array}{c}-0.017^{* * *} \\
(-3.318)\end{array}$ & $\begin{array}{l}-0.016^{*} \\
(-1.705)\end{array}$ & $\begin{array}{c}-0.033^{* * *} \\
(-2.993)\end{array}$ \\
\hline $\ln E S_{2}$ & $\begin{array}{l}0.008^{* *} \\
(2.033)\end{array}$ & $\begin{array}{l}0.018^{* *} \\
(2.472)\end{array}$ & $\begin{array}{c}0.026^{* * * *} \\
(2.928)\end{array}$ & $\begin{array}{l}0.009 \text { ** } \\
(2.109)\end{array}$ & $\begin{array}{c}0.018^{* *} \\
(2.512)\end{array}$ & $\begin{array}{c}0.026^{* * * *} \\
(2.959)\end{array}$ \\
\hline $\ln E R_{1}$ & $\begin{array}{l}-0.010^{*} \\
(-1.751)\end{array}$ & $\begin{array}{c}-0.007 \\
(-0.703)\end{array}$ & $\begin{array}{c}-0.017 \\
(-1.329)\end{array}$ & $\begin{array}{l}-0.010^{*} \\
(-1.754)\end{array}$ & $\begin{array}{c}-0.005 \\
(-0.447)\end{array}$ & $\begin{array}{c}-0.015 \\
(-1.123)\end{array}$ \\
\hline $\ln E R_{2}$ & $\begin{array}{l}0.041 * \\
(1.886)\end{array}$ & $\begin{array}{c}-0.040 \\
(-0.850)\end{array}$ & $\begin{array}{c}0.001 \\
(0.009)\end{array}$ & $\begin{array}{l}0.040 * \\
(1.831)\end{array}$ & $\begin{array}{c}-0.039 \\
(-0.787)\end{array}$ & $\begin{array}{c}0.000 \\
(0.002)\end{array}$ \\
\hline $\ln L F$ & $\begin{array}{l}0.006^{* *} \\
(2.053)\end{array}$ & $\begin{array}{c}-0.001 \\
(-0.183)\end{array}$ & $\begin{array}{c}0.005 \\
(0.859)\end{array}$ & $\begin{array}{l}0.006^{* *} \\
(2.051)\end{array}$ & $\begin{array}{c}0.001 \\
(0.158)\end{array}$ & $\begin{array}{c}0.007 \\
(1.111)\end{array}$ \\
\hline $\ln F D$ & $\begin{array}{c}-0.007 \\
(-0.825)\end{array}$ & $\begin{array}{c}-0.010 \\
(-0.711)\end{array}$ & $\begin{array}{c}-0.018 \\
(-1.069)\end{array}$ & $\begin{array}{c}-0.007 \\
(-0.767)\end{array}$ & $\begin{array}{c}-0.009 \\
(-0.637)\end{array}$ & $\begin{array}{c}-0.016 \\
(-0.958)\end{array}$ \\
\hline $\ln H C$ & $\begin{array}{c}0.040^{* * *} \\
(5.446)\end{array}$ & $\begin{array}{c}-0.010 \\
(-0.818)\end{array}$ & $\begin{array}{l}0.030 * * \\
(2.257)\end{array}$ & $\begin{array}{c}0.040^{* * *} \\
(5.444)\end{array}$ & $\begin{array}{c}-0.009 \\
(-0.762)\end{array}$ & $\begin{array}{c}0.031 * * \\
(2.226)\end{array}$ \\
\hline $\ln F D I$ & $\begin{array}{l}0.007^{* *} \\
(2.314)\end{array}$ & $\begin{array}{c}-0.006 \\
(-1.566)\end{array}$ & $\begin{array}{c}0.001 \\
(0.195)\end{array}$ & $\begin{array}{l}0.007^{* *} \\
(2.281)\end{array}$ & $\begin{array}{c}-0.006 \\
(-1.293)\end{array}$ & $\begin{array}{c}0.001 \\
(0.254)\end{array}$ \\
\hline $\ln I U$ & $\begin{array}{l}0.034^{* *} \\
(2.155)\end{array}$ & $\begin{array}{c}-0.033 \\
(-1.155)\end{array}$ & $\begin{array}{c}0.001 \\
(0.034)\end{array}$ & $\begin{array}{l}0.036^{* *} \\
(2.227)\end{array}$ & $\begin{array}{c}-0.034 \\
(-1.172)\end{array}$ & $\begin{array}{c}0.002 \\
(0.062)\end{array}$ \\
\hline
\end{tabular}

Notes: The t-statistics are given in the parentheses; ${ }^{* * *}, * *$, and ${ }^{*}$ denote statistical significance at the $1 \%, 5 \%$, and $10 \%$ significance levels, respectively.

Table 8 reports the direct, indirect and total effects of the central region. As is shown in Table 8 , the coefficients of direct effect and indirect effect are nearly consistent with the corresponding coefficients in Table 6, indicating that the spatial feedback effects among central cities are also negligible. The total effect of $\ln E R_{2}$ is significantly and positively associated with $\ln U D Q$, while other variables are not statistically significant, implying the importance of the removal rate of smoke and dust on urban development quality in the central region. It is noteworthy that most of the coefficients are not significant in the central region. One possible reason is that the intensity of air pollution control in the central region is relatively low compared to that in the eastern region. Besides, the local governments in the central region generally lack awareness of environment protection and do not have sufficient supervision on the implement of air pollution control. 
Table 8. The direct, indirect and total effects of the central region.

\begin{tabular}{|c|c|c|c|c|c|c|}
\hline \multirow{2}{*}{ Variables } & \multicolumn{3}{|c|}{$w_{i j}^{1}$} & \multicolumn{3}{|c|}{$w_{i j}^{2}$} \\
\hline & Direct Effect & Indirect Effect & Total Effect & Direct Effect & Indirect Effect & Total Effect \\
\hline $\ln E S_{1}$ & $\begin{array}{l}-0.006 \\
(-0.582)\end{array}$ & $\begin{array}{c}0.022 \\
(1.463)\end{array}$ & $\begin{array}{c}0.016 \\
(0.936)\end{array}$ & $\begin{array}{c}-0.005 \\
(-0.467) \\
\end{array}$ & $\begin{array}{c}0.013 \\
(0.851)\end{array}$ & $\begin{array}{c}0.008 \\
(0.494)\end{array}$ \\
\hline $\ln E S_{2}$ & $\begin{array}{c}0.008 \\
(1.481)\end{array}$ & $\begin{array}{c}0.002 \\
(0.221)\end{array}$ & $\begin{array}{c}0.010 \\
(0.974)\end{array}$ & $\begin{array}{c}0.009 \\
(1.534)\end{array}$ & $\begin{array}{c}0.005 \\
(0.582)\end{array}$ & $\begin{array}{c}0.014 \\
(1.334)\end{array}$ \\
\hline $\ln E R_{1}$ & $\begin{array}{c}-0.002 \\
(-0.436)\end{array}$ & $\begin{array}{c}-0.009 \\
(-1.236)\end{array}$ & $\begin{array}{l}-0.011 \\
(-1.274)\end{array}$ & $\begin{array}{c}-0.002 \\
(-0.413)\end{array}$ & $\begin{array}{c}-0.010 \\
(-1.301)\end{array}$ & $\begin{array}{c}-0.012 \\
(-1.329) \\
\end{array}$ \\
\hline $\ln E R_{2}$ & $\begin{array}{c}0.018 \\
(0.873)\end{array}$ & $\begin{array}{c}0.060 \text { ** } \\
(2.149)\end{array}$ & $\begin{array}{l}0.079^{* *} \\
(2.217)\end{array}$ & $\begin{array}{c}0.016 \\
(0.764)\end{array}$ & $\begin{array}{l}0.059^{*} \\
(1.778)\end{array}$ & $\begin{array}{l}0.075^{*} \\
(1.900)\end{array}$ \\
\hline $\ln L F$ & $\begin{array}{c}0.003 \\
(0.995)\end{array}$ & $\begin{array}{c}-0.005 \\
(-1.000)\end{array}$ & $\begin{array}{l}-0.002 \\
(-0.258)\end{array}$ & $\begin{array}{c}0.003 \\
(1.054)\end{array}$ & $\begin{array}{c}-0.003 \\
(-0.683)\end{array}$ & $\begin{array}{c}0.000 \\
(0.016)\end{array}$ \\
\hline $\ln F D$ & $\begin{array}{c}0.009 \\
(0.813)\end{array}$ & $\begin{array}{l}-0.008 \\
(-0.426)\end{array}$ & $\begin{array}{c}0.001 \\
(0.0600)\end{array}$ & $\begin{array}{c}0.007 \\
(0.633)\end{array}$ & $\begin{array}{l}-0.001 \\
(-0.083)\end{array}$ & $\begin{array}{c}0.006 \\
(0.292)\end{array}$ \\
\hline $\ln H C$ & $\begin{array}{l}0.021^{* *} \\
(2.448) \\
\end{array}$ & $\begin{array}{c}-0.036^{* * *} \\
(-3.029) \\
\end{array}$ & $\begin{array}{c}-0.015 \\
(-1.072) \\
\end{array}$ & $\begin{array}{l}0.020^{* *} \\
(2.475) \\
\end{array}$ & $\begin{array}{c}-0.035^{* * *} \\
(-2.931) \\
\end{array}$ & $\begin{array}{c}-0.014 \\
(-1.053) \\
\end{array}$ \\
\hline $\ln F D I$ & $\begin{array}{c}-0.002 \\
(-0.685)\end{array}$ & $\begin{array}{c}-0.004 \\
(-1.033)\end{array}$ & $\begin{array}{l}-0.005 \\
(-1.436)\end{array}$ & $\begin{array}{l}-0.002 \\
(-0.613)\end{array}$ & $\begin{array}{l}-0.004 \\
(-1.122)\end{array}$ & $\begin{array}{c}-0.005 \\
(-1.460) \\
\end{array}$ \\
\hline $\ln I U$ & $\begin{array}{c}0.012 \\
(0.812)\end{array}$ & $\begin{array}{l}-0.033 \\
(-1.439)\end{array}$ & $\begin{array}{l}-0.021 \\
(-0.840)\end{array}$ & $\begin{array}{c}0.013 \\
(0.884)\end{array}$ & $\begin{array}{l}-0.034 \\
(-1.537)\end{array}$ & $\begin{array}{l}-0.021 \\
(-0.851)\end{array}$ \\
\hline
\end{tabular}

Notes: The t-statistics are given in the parentheses; ${ }^{* * *}, * *$, and ${ }^{*}$ denote statistical significance at the $1 \%, 5 \%$, and $10 \%$ significance levels, respectively.

Table 9 reports the direct, indirect and total effects of the western region. As is shown in Table 9, the coefficients of direct effect and indirect effect are nearly consistent with the corresponding coefficients in Table 6, indicating that the spatial feedback effects among western cities are also negligible. The total effect of $\ln E R_{1}$ is significantly and positively associated with $\ln U D Q$, while other variables are not statistically significant, implying the importance of the intensity of sulfur dioxide on urban development quality in the western region. Similar to the central region, the intensity of air pollution control in the western region is relatively low compared to that in the eastern region. Besides, the local governments in the western region also lack consciousness of environment protection and do not have sufficient supervision on the implement of air pollution control.

Table 9. The direct, indirect and total effects of the western region.

\begin{tabular}{|c|c|c|c|c|c|c|}
\hline \multirow{2}{*}{ Variables } & \multicolumn{3}{|c|}{$w_{i j}^{1}$} & \multicolumn{3}{|c|}{$w_{i j}^{2}$} \\
\hline & Direct Effect & Indirect Effect & Total Effect & Direct Effect & Indirect Effect & Total Effect \\
\hline $\ln E S_{1}$ & $\begin{array}{c}-0.003 \\
(-0.718)\end{array}$ & $\begin{array}{c}0.005 \\
(0.701)\end{array}$ & $\begin{array}{c}0.001 \\
(0.168)\end{array}$ & $\begin{array}{c}-0.003 \\
(-0.778)\end{array}$ & $\begin{array}{c}0.006 \\
(0.898)\end{array}$ & $\begin{array}{c}0.003 \\
(0.328)\end{array}$ \\
\hline $\ln E S_{2}$ & $\begin{array}{c}0.002 \\
(0.554)\end{array}$ & $\begin{array}{c}0.005 \\
(0.965)\end{array}$ & $\begin{array}{c}0.006 \\
(1.089)\end{array}$ & $\begin{array}{c}0.001 \\
(0.482)\end{array}$ & $\begin{array}{c}0.005 \\
(0.976)\end{array}$ & $\begin{array}{c}0.006 \\
(1.049)\end{array}$ \\
\hline $\ln E R_{1}$ & $\begin{array}{c}0.003 \\
(0.961)\end{array}$ & $\begin{array}{l}0.008 * \\
(1.940)\end{array}$ & $\begin{array}{c}0.010^{* *} \\
(2.127)\end{array}$ & $\begin{array}{c}0.002 \\
(0.927)\end{array}$ & $\begin{array}{l}0.007^{*} \\
(1.784)\end{array}$ & $\begin{array}{l}0.010^{*} \\
(1.939)\end{array}$ \\
\hline $\ln E R_{2}$ & $\begin{array}{c}-0.008 \\
(-1.018)\end{array}$ & $\begin{array}{c}-0.014 \\
(-1.084)\end{array}$ & $\begin{array}{c}-0.022 \\
(-1.379)\end{array}$ & $\begin{array}{c}-0.007 \\
(-0.993)\end{array}$ & $\begin{array}{c}-0.010 \\
(-0.789)\end{array}$ & $\begin{array}{c}-0.017 \\
(-1.162)\end{array}$ \\
\hline $\ln L F$ & $\begin{array}{c}0.004^{* *} \\
(2.422)\end{array}$ & $\begin{array}{c}0.002 \\
(0.530)\end{array}$ & $\begin{array}{l}0.006^{*} \\
(1.723)\end{array}$ & $\begin{array}{l}0.004^{* *} \\
(2.371)\end{array}$ & $\begin{array}{c}0.001 \\
(0.364)\end{array}$ & $\begin{array}{c}0.005 \\
(1.531)\end{array}$ \\
\hline $\ln F D$ & $\begin{array}{c}-0.005 \\
(-0.714)\end{array}$ & $\begin{array}{c}0.003 \\
(0.395)\end{array}$ & $\begin{array}{c}-0.002 \\
(-0.214)\end{array}$ & $\begin{array}{c}-0.004 \\
(-0.634)\end{array}$ & $\begin{array}{c}0.003 \\
(0.304)\end{array}$ & $\begin{array}{c}-0.002 \\
(-0.226)\end{array}$ \\
\hline $\ln H C$ & $\begin{array}{c}-0.001 \\
(-0.335)\end{array}$ & $\begin{array}{c}0.004 \\
(0.994)\end{array}$ & $\begin{array}{c}0.003 \\
(0.676)\end{array}$ & $\begin{array}{c}-0.001 \\
(-0.268)\end{array}$ & $\begin{array}{c}0.004 \\
(0.891)\end{array}$ & $\begin{array}{c}0.003 \\
(0.627)\end{array}$ \\
\hline $\ln F D I$ & $\begin{array}{c}0.001 \\
(0.955)\end{array}$ & $\begin{array}{c}0.000 \\
(0.295)\end{array}$ & $\begin{array}{c}0.001 \\
(0.810)\end{array}$ & $\begin{array}{c}0.001 \\
(1.018)\end{array}$ & $\begin{array}{c}0.001 \\
(0.399)\end{array}$ & $\begin{array}{c}0.002 \\
(0.902)\end{array}$ \\
\hline $\ln I U$ & $\begin{array}{c}0.007 \\
(0.910)\end{array}$ & $\begin{array}{c}-0.006 \\
(-0.569)\end{array}$ & $\begin{array}{c}0.001 \\
(0.049)\end{array}$ & $\begin{array}{c}0.008 \\
(0.977)\end{array}$ & $\begin{array}{c}-0.001 \\
(-0.071)\end{array}$ & $\begin{array}{c}0.007 \\
(0.472)\end{array}$ \\
\hline
\end{tabular}

Notes: The t-statistics are given in the parentheses; ${ }^{* *}$ and ${ }^{*}$ denote statistical significance at the $5 \%$ and $10 \%$ significance levels, respectively. 


\section{Conclusions and Policy Implications}

With the panel data of 285 prefecture-level cities in China from 2003-2016, an index of urban development quality is constructed and calculated in this paper, based on the combination of principal component analysis and efficacy coefficient method. Through the application of spatial Durbin model, and under the framework of unified analysis, the role and mechanism of air pollution control on the impact of urban development quality are investigated. Three main conclusions can be drawn from the above analysis. First, during the investigation, the intensity of air pollution control and urban development quality have been enhanced, however, different air pollution control, especially the decline of sulfur dioxide, which erodes urban development quality at the national level, do not all play a positive role in improving the urban development quality as expected. Second, the impact of different types of air pollution control on urban development quality varies from region to region. In the eastern region, the direct effect of $\ln E S_{1}$ and $\ln E R_{1}$ is significantly and negatively correlated with urban development quality while the direct effect of $\ln E S_{2}$ and $\ln E R_{2}$ is significantly and positively associated with urban development quality, indicating that the control of smoke and dust has improved urban development quality, while the control of sulfur dioxide is at the sacrifice of the deterioration of urban development quality. In the central and the western regions, the direct effect of air pollution control on urban development quality does not pass the test of significance, indicating that the local government generally lacks consciousness of environment protection and does not have sufficient supervision of the implement of air pollution control compared with the local government in the eastern region. Third, the spatial coefficients $(\rho)$ are also highly significant in China, the eastern region and the western region, which is a strong evidence of spatial dependence. However, it does not pass the test of significance in the central region, implying the spatial dependence of the central region is weak and poor compared with that of the eastern and western regions.

Three important policy implications can be drawn from the above conclusions. First, to comprehensively and thoroughly realize the goal of environmental protection and the improvement of urban development quality, it is necessary to promote the structural upgrading of the energy system. On the whole, clean energy should gradually replace fossil energy, for the reduction of smoke and dust will improve the urban development quality. However, if the alternative energy is insufficient, unreasonably pursuing the reduction of sulfur dioxide will hinder the improvement of urban development quality. Therefore, promoting the structural upgrading of the energy system is the most fundamental solution. Second, the local government should transform the excessive pursuit of short-term economic growth, and strive to improve the urban development quality comprehensively. Due to the lack of the consciousness of environment protection and the sufficient supervision of the implement of air pollution control, the impact of air pollution control on urban development quality in the central and western regions does not pass the test of significance, therefore, it is necessary and urgent to transform the reliance on extensive development models, and raise the awareness of environmental protection and sustainable development, and ultimately promote the improvement of urban development quality in the long run. Third, the links of environmental protection should be strengthened between different regions. Therefore, strengthening the legal basis of emission trading, compensation mechanisms and enforcement within the region and more importantly between regions can guarantee consistency and fairness of air pollution control policies, and ensure that the interests of urban development quality of inter-regional cities can be reasonably balanced.

Although this study provides valuable insights, it has three limitations, which should serve to stimulate further research. First, due to data restrictions, the period covered in this study is only fourteen years. To confirm our findings, the time span can be increased to cover a longer period, and more information and data can be used for comprehensive and thorough analysis. Second, in our study, air pollution control is divided into two types based on the difference before and after the treatment, and each type of air pollution control is measured by two typical indicators in empirical research. In further research, an expansion of the indicator system may be considered to obtain more guiding conclusions. Third, the spatial Durbin model is adopted to do the empirical analysis in this 
paper, but time effect is ignored, so the results may have some deviations compared to the actual situation. To expand the research, the dynamic spatial Durbin model should be adopted to empirically study the impact of air pollution control on urban development quality in China and other developing countries undergoing similar urbanization and modernization processes.

Author Contributions: Y.F. had the initial idea for the study and interpreted the results. X.W. designed the conceptual framework of the methodology, drafted and revised the manuscript. W.D. is responsible for the data collection and econometric analysis. J.L. performed the calculations and analyzed the data. All of the authors have contributed to the writing and finalizing of the paper.

Funding: This research is supported by the National Natural Science Foundation of China (No. 71473057 and No. 71874042).

Acknowledgments: The authors wish to thank the three anonymous reviewers for valuable comments and constructive suggestions on the early drafts of this paper. Any remaining errors are the responsibility of the authors.

Conflicts of Interest: The authors declare no conflict of interest.

\section{Appendix A. Lists of 285 Prefecture-Level Cities Included into the Sample}

Table A1. The 285 prefecture-level cities.

\begin{tabular}{|c|c|c|c|c|c|c|c|c|}
\hline \multicolumn{3}{|c|}{ Eastern City (101) } & \multicolumn{3}{|c|}{ Central City (100) } & \multicolumn{3}{|c|}{ Western City (84) } \\
\hline Beijing & Lianyungang & Jining & Taiyuan & Huainan & Luohe & Hohhot & Nanchong & Tianshui \\
\hline Tianjin & Huai'an & Tai'an & Datong & Maanshan & Sanmenxia & Baotou & Meishan & Wuwei \\
\hline Shijiazhuang & Yancheng & Weihai & Yangquan & Huaibei & Nanyang & Wuhai & Yibin & Zhangye \\
\hline Tangshan & Yangzhou & Rizhao & Changzhi & Tongling & Shangqiu & Chifeng & Guang'an & Pingliang \\
\hline Qinhuangdao & Zhenjiang & Laiwu & Jincheng & Anqing & Xinyang & Tongliao & Dazhou & Jiuquan \\
\hline Handan & Taizhou & Linyi & Shuozhou & Huangshan & Zhoukou & Erdos & Ya'an & Qingyang \\
\hline Xingtai & Suqian & Dezhou & Jinzhong & Chuzhou & Zhumadian & Hulunbuir & Bazhong & Dingxi \\
\hline Baoding & Hangzhou & Liaocheng & Yuncheng & Fuyang & Wuhan & Bayannur & Ziyang & Longnan \\
\hline Zhangiiakou & Ningbo & Binzhou & Xinzhou & Suzhou & Huangshi & Ulangab & Guiyang & Xining \\
\hline Chengde & Wenzhou & Heze & Linfen & Lu'an & Shiyan & Nanning & Liupanshui & Yinchuan \\
\hline Cangzhou & Jiaxing & Guangzhou & Lvliang & Bozhou & Yichang & Liuzhou & Zunyi & Shizuishan \\
\hline Langfang & Huzhou & Shaoguan & Changchun & Chizhou & Xiangyang & Guilin & Anshun & Wuzhong \\
\hline Hengshui & Shaoxing & Shenzhen & Jilin & Xuancheng & Ezhou & Wuzhou & Kunming & Guyuan \\
\hline Shenyang & Jinhua & Zhuhai & Siping & Nanchang & Jingmen & Beihai & Qujing & Zhongwei \\
\hline Dalian & Quzhou & Shantou & Liaoyuan & Jingdezhen & Xiaogan & Fangchenggang & Yuxi & Urumgi \\
\hline Anshan & Zhoushan & Foshan & Tonghua & Pingxiang & Jingzhou & Qinzhou & Baoshan & Karamay \\
\hline Fushun & Taizhou & Jiangmen & Baishan & Jiujiang & Huanggang & Guigang & Zhaotong & \\
\hline Benxi & Lishui & Zhanjiang & Songyuan & Xinyu & Xianning & Yulin & Lijiang & \\
\hline Dandong & Fuzhou & Maoming & Baicheng & Yingtan & Suizhou & Baise & $\begin{array}{l}\text { Py'er } \\
\text { Pu's }\end{array}$ & \\
\hline Jinzhou & Xiamen & Zhaoqing & Harbin & Ganzhou & Changsha & Hezhou & Lincang & \\
\hline Yingkou & Putian & Huizhou & Qiqihar & Ji'an & Zhuzhou & Hechi & Xi'an & \\
\hline Fuxin & Sanming & Meizhou & Jixi & Yichun & Xiangtan & Laibin & Tongchuan & \\
\hline Liaoyang & Quanzhou & Shanwei & Hegang & Fuzhou & Hengyang & Chongzuo & Baoji & \\
\hline Panjin & Zhangzhou & Heyuan & Shuangyashan & Shangrao & Shaoyang & Chongqing & Xianyang & \\
\hline Tiding & Nanping & Yangjiang & Daqing & Zhengzhou & Yueyang & Chengdu & Weinan & \\
\hline Chaoyang & Longyan & Qingyuan & Yichun & Kaifeng & Changde & Zigong & Yan'an & \\
\hline Huludao & Ningde & Dongguan & Jiamusi & Luoyang & Zhangiiajie & Panzhihua & Hanzhong & \\
\hline Shanghai & Jinan & Zhongshan & Qitaihe & Pingdingshan & Yiyang & Luzhou & Yulin & \\
\hline Nanjing & Qingdao & Chaozhou & Mudanjiang & Anyang & Chenzhou & Deyang & Ankang & \\
\hline Wuxi & Zibo & Jieyang & Heihe & Hebi & Yongzhou & Mianyang & Shangluo & \\
\hline Xuzhou & Zaozhuang & Yunfu & Suihua & Xinxiang & Huaihua & Guangyuan & Lanzhou & \\
\hline Changzhou & Dongying & Haikou & Hefei & Jiaozuo & Loudi & Suining & Jiayuguan & \\
\hline Suzhou & Yantai & Sanya & Wuhu & Puyang & & Neijiang & Jinchang & \\
\hline Nantong & Weifang & & Bengbu & Xuchang & & Leshan & Baiyin & \\
\hline
\end{tabular}




\section{Appendix B. Data Sources}

Table A2. Data Sources.

\begin{tabular}{|c|c|c|c|}
\hline Variable & Classification & Composition & Sources \\
\hline \multirow{9}{*}{$\begin{array}{l}\text { Urban } \\
\text { development } \\
\text { quality }\end{array}$} & Economic dimension & $\begin{array}{c}\text { The proportion of secondary } \\
\text { industries' employee } \\
\text { The proportion of tertiary industries' employee } \\
\text { Per capita GDP } \\
\text { per capita total retail sales of consumer goods } \\
\text { Per capita education funding }\end{array}$ & $\begin{array}{l}\text { China City Statistical Yearbook } \\
\text { China City Statistical Yearbook } \\
\text { China City Statistical Yearbook } \\
\text { China City Statistical Yearbook } \\
\text { China City Statistical Yearbook }\end{array}$ \\
\hline & \multirow{4}{*}{ Social dimension } & Employment density & $\begin{array}{c}\text { China Urban Construction Statistical } \\
\text { Yearbook } \\
\text { China Urban Construction Statistical } \\
\text { Yearbook }\end{array}$ \\
\hline & & $\begin{array}{l}\text { Urban construction land accounting for the } \\
\text { proportion of urban areas }\end{array}$ & China City Statistical Yearbook \\
\hline & & Green area coverage in built-up areas & $\begin{array}{c}\text { China Urban Construction Statistical } \\
\text { Yearbook }\end{array}$ \\
\hline & & $\begin{array}{l}\text { Investment completion of per capita real } \\
\text { estate development }\end{array}$ & China City Statistical Yearbook \\
\hline & \multirow{4}{*}{$\begin{array}{l}\text { Environmental } \\
\text { dimension }\end{array}$} & $\begin{array}{l}\text { Comprehensive utilization rate of industrial } \\
\text { solid waste }\end{array}$ & China City Statistical Yearbook \\
\hline & & $\begin{array}{l}\text { Sewage centralized treatment rate } \\
\text { Harmless treatment rate of domestic garbage }\end{array}$ & $\begin{array}{l}\text { China City Statistical Yearbook } \\
\text { China City Statistical Yearbook }\end{array}$ \\
\hline & & Number of public toilets & $\begin{array}{c}\text { China Urban Construction Statistical } \\
\text { Yearbook }\end{array}$ \\
\hline & & Per capita public green area & $\begin{array}{c}\text { China Urban Construction Statistical } \\
\text { Yearbook }\end{array}$ \\
\hline \multirow{2}{*}{$\begin{array}{l}\text { Environmental } \\
\text { regulation }\end{array}$} & $\begin{array}{l}\text { Energy-saving } \\
\text { regulation }\end{array}$ & $\begin{array}{l}\text { The emission of sulfur dioxide per GDP } \\
\text { The emission of smoke and dust per GDP }\end{array}$ & $\begin{array}{l}\text { China City Statistical Yearbook } \\
\text { China City Statistical Yearbook }\end{array}$ \\
\hline & $\begin{array}{l}\text { Emission-reduction } \\
\text { regulation }\end{array}$ & $\begin{array}{l}\text { The removal rate of sulfur dioxide } \\
\text { The removal rate of smoke and dust }\end{array}$ & $\begin{array}{l}\text { China City Statistical Yearbook } \\
\text { China City Statistical Yearbook }\end{array}$ \\
\hline \multirow{5}{*}{ Control variables } & Land finance & The shares of land leasing revenue in GDP & China Land and Resources Almanac \\
\hline & Finance development & The shares of both deposits and loans in GDP & China City Statistical Yearbook \\
\hline & Human capital & The number of college students per 10,000 people & China City Statistical Yearbook \\
\hline & $\begin{array}{l}\text { Foreign direct } \\
\text { investment }\end{array}$ & The shares of foreign direct investment in GDP & China City Statistical Yearbook \\
\hline & Industrial upgrading & $\begin{array}{l}\text { The shares of the value of the tertiary industries } \\
\text { in the value of the secondary industries }\end{array}$ & China City Statistical Yearbook \\
\hline
\end{tabular}

\section{References}

1. Wang, L.; Zhang, F.Y.; Pilot, E.; Yu, J.; Nie, C.J.; Holdaway, J.; Yang, L.S.; Li, Y.H.; Wang, W.Y.; Vardoulakis, S.; et al. Taking Action on Air Pollution Control in the Beijing-Tianjin-Hebei (BTH) Region: Progress, Challenges and Opportunities. Int. J. Environ. Res. Public Health 2018, 15, 306. [CrossRef] [PubMed]

2. Wei, Y.D.; Ewing, R. Urban expansion, sprawl and inequality. Landsc. Urban Plan. 2018, 177, $259-265$. [CrossRef]

3. Hao, Y.; Deng, Y.X.; Lu, Z.N.; Chen, H. Is environmental regulation effective in China? Evidence from city-level panel data. J. Clean. Prod. 2018, 188, 966-976. [CrossRef]

4. Farinmade, A.; Soyinka, O.; Siu, K.W.M. Assessing the effect of urban informal economic activity on the quality of the built environment for sustainable urban development in Lagos, Nigeria. Sustain. Cities Soc. 2018, 41, 13-21. [CrossRef]

5. Metzger, J.; Wiberg, S. Contested framings of urban qualities: Dis/qualifications of value in urban development controversies. Urban Stud. 2018, 55, 2300-2316. [CrossRef]

6. Cahantimur, A.; Ozturk, R.B.; Erkan, B.H. Healthy and Quality Urban Spaces for Sustainable development of Heritage Sites. Eur. J. Sustain. Dev. 2018, 7, 317-328. [CrossRef]

7. Wang, C.N.; Nguyen, H.K. Enhancing Urban Development Quality Based on the Results of Appraising Efficient Performance of Investors-A Case Study in Vietnam. Sustainability 2017, 9, 1397. [CrossRef]

8. $\quad$ Long, Y.; Zhai, W.X.; Shen, Y.; Ye, X.Y. Understanding uneven urban expansion with natural cities using open data. Landsc. Urban Plan. 2018, 177, 281-293. [CrossRef] 
9. Mao, D.H.; Wang, Z.M.; Wu, J.G.; Wu, B.F.; Zeng, Y.; Song, K.S.; Yi, K.P.; Luo, L. China's wetlands loss to urban expansion. Land Degrad. Dev. 2018, 29, 2644-2657. [CrossRef]

10. Zhang, Z.X.; Liu, F.; Zhao, X.L.; Wang, X.; Shi, L.F.; Xu, J.Y.; Yu, S.S.; Wen, Q.K.; Zuo, L.J.; Yi, L.; et al. Urban Expansion in China Based on Remote Sensing Technology: A Review. Chin. Geogr. Sci. 2018, 28, 727-743. [CrossRef]

11. Wang, T.; Yang, M.H. Land Use and Land Cover Change in China's Loess Plateau: The Impacts of Climate Change, Urban Expansion and Grain for Green Project Implementation. Appl. Ecol. Env. Res. 2018, 16, 4145-4163. [CrossRef]

12. Gong, J.Z.; Hu, Z.R.; Chen, W.L.; Liu, Y.S.; Wang, J.Y. Urban expansion dynamics and modes in metropolitan Guangzhou, China. Land Use Policy 2018, 72, 100-109. [CrossRef]

13. Cao, Q.; Yu, D.Y.; Georgescu, M.; Wu, J.G.; Wang, W. Impacts of future urban expansion on summer climate and heat-related human health in eastern China. Environ. Int. 2018, 112, 134-146. [CrossRef] [PubMed]

14. Liu, Y.; Fan, P.L.; Yue, W.Z.; Song, Y. Impacts of land finance on urban sprawl in China: The case of Chongqing. Land Use Policy 2018, 72, 420-432. [CrossRef]

15. Li, S.Y.; Liu, X.P.; Li, Z.G.; Wu, Z.F.; Yan, Z.J.; Chen, Y.M.; Gao, F. Spatial and Temporal Dynamics of Urban Expansion along the Guangzhou-Foshan Inter-City Rail Transit Corridor, China. Sustainability 2018, 10, 593. [CrossRef]

16. Wei, Y.H.D.; Li, H.; Yue, W.Z. Urban land expansion and regional inequality in transitional China. Landsc. Urban Plan. 2017, 163, 17-31. [CrossRef]

17. Li, H.; Zhang, J.X.; Wang, C.; Wang, Y.J.; Coffey, V. An evaluation of the impact of environmental regulation on the efficiency of technology innovation using the combined DEA model: A case study of Xi'an, China. Sustain. Cities Soc. 2018, 42, 355-369. [CrossRef]

18. Taylor, C.M.; Gallagher, E.A.; Pollard, S.J.T.; Rocks, S.A.; Smith, H.M.; Leinster, P.; Angus, A.J. Environmental regulation in transition: Policy officials' views of regulatory instruments and their mapping to environmental risks. Sci. Total Environ. 2019, 646, 811-820. [CrossRef]

19. Kostecki, P.T.; Calabrese, E.J. The Regulation of Petroleum Contaminated Soils-a Question of Environmental and Public-Health Effects. J. Am. Coll. Toxicol. 1985, 4, 373.

20. Iwama, G.K.; Heisler, N. Effect of Environmental Water Salinity on Acid-Base Regulation during Environmental Hypercapnia in the Rainbow-Trout (Oncorhynchus-Mykiss). J. Exp. Biol. 1991, 158, 1-18.

21. Ajuzie, E.; Altobello, M. Effects of environmental pollution and public regulations on productivity growth in the Long Island Sound oyster industry: A dual translog cost function approach. Am. J. Agric. Econ. 1995, 77,1386 .

22. Lee, M. The effect of environmental regulations: A restricted cost function for Korean manufacturing industries. Environ. Dev. Econ. 2007, 12, 91-104. [CrossRef]

23. Yu, Y.T.; Huang, J.H.; Luo, N.S. Can More Environmental Information Disclosure Lead to Higher Eco-efficiency? Evidence from China. Sustainability 2018, 10, 528.

24. Feng, Z.J.; Chen, W. Environmental Regulation, Green Innovation, and Industrial Green Development: An Empirical Analysis Based on the Spatial Durbin Model. Sustainability 2018, 10, 223. [CrossRef]

25. Kirkpatrick, C.; Shimamoto, K. The effect of environmental regulation on the locational choice of Japanese foreign direct investment. Appl. Econ. 2008, 40, 1399-1409. [CrossRef]

26. He, H.R. Effects of environmental policy on consumption: Lessons from the Chinese plastic bag regulation. Environ. Dev. Econ. 2012, 17, 407-431. [CrossRef]

27. Zhao, L.S.; Sun, C.Z.; Liu, F.C. Interprovincial two-stage water resource utilization efficiency under environmental constraint and spatial spillover effects in China. J. Clean. Prod. 2017, 164, 715-725. [CrossRef]

28. LeSage, J.P. Spatial econometric panel data model specification: A Bayesian approach. Spat. Stat. 2014, 9, 38-145. [CrossRef]

29. LeSage, J.P.; Sheng, Y.X. A spatial econometric panel data examination of endogenous versus exogenous interaction in Chinese province-level patenting. J. Geogr. Syst. 2014, 16, 233-262. [CrossRef]

30. Elhorst, J.P. Applied Spatial Econometrics: Raising the Bar. Spat. Econ. Anal. 2010, 5, 9-28. [CrossRef]

31. Elhorst, J.P. Dynamic spatial panels: Models, methods, and inferences. J. Geogr. Syst. 2012, 14, 5-28. [CrossRef]

32. Bera, A.K.; Dogan, O.; Taspinar, S. Simple tests for endogeneity of spatial weights matrices. Reg. Sci. Urban Econ. 2018, 69, 130-142. [CrossRef] 
33. Liu, Z.; Liu, S.H.; Qi, W.; Jin, H.R. Urban sprawl among Chinese cities of different population sizes. Habitat Int. 2018, 79, 89-98. [CrossRef]

34. Dunea, D.; Iordache, S.; Liu, H.Y.; Bohler, T.; Pohoata, A.; Radulescu, C. Quantifying the impact of $\mathrm{PM}_{2.5}$ and associated heavy metals on respiratory health of children near metallurgical facilities. Environ. Sci. Pollut. Res. 2016, 23, 15395-15406. [CrossRef] [PubMed]

35. Chen, R.; Ju, M.T.; Chu, C.L.; Jing, W.Q.; Wang, Y.Q. Identification and Quantification of Physicochemical Parameters Influencing Chlorophyll-a Concentrations through Combined Principal Component Analysis and Factor Analysis: A Case Study of the Yuqiao Reservoir in China. Sustainability 2018, 10, 936. [CrossRef]

36. Levin, A.; Lin, C.F.; Chu, C.S.J. Unit root tests in panel data: Asymptotic and finite-sample properties. J. Econ. 2002, 108, 1-24. [CrossRef]

37. Im, K.S.; Pesaran, M.H.; Shin, Y. Testing for unit roots in heterogeneous panels. J. Econ. 2003, 115, 53-74. [CrossRef]

38. Maddala, G.S.; Wu, S.W. A comparative study of unit root tests with panel data and a new simple test. Oxf. Bull. Econ. Stat. 1999, 61, 631-652. [CrossRef]

(C) 2018 by the authors. Licensee MDPI, Basel, Switzerland. This article is an open access article distributed under the terms and conditions of the Creative Commons Attribution (CC BY) license (http://creativecommons.org/licenses/by/4.0/). 